ESAIM: COCV 19 (2013) 358-384

DOI: $10.1051 / \mathrm{cocv} / 2012012$
ESAIM: Control, Optimisation and Calculus of Variations

www.esaim-cocv.org

\title{
WELL-POSEDNESS OF A CLASS OF NON-HOMOGENEOUS BOUNDARY VALUE PROBLEMS OF THE KORTEWEG-DE VRIES EQUATION ON A FINITE DOMAIN*,**
}

\author{
Eugene Kramer ${ }^{1}$, Ivonne Rivas ${ }^{2,3}$ and Bing-Yu Zhang ${ }^{2}$
}

\begin{abstract}
In this paper, we study a class of Initial-Boundary Value Problems proposed by Colin and Ghidaglia for the Korteweg-de Vries equation posed on a bounded domain $(0, L)$. We show that this class of Initial-Boundary Value Problems is locally well-posed in the classical Sobolev space $H^{s}(0, L)$ for $s>-\frac{3}{4}$, which provides a positive answer to one of the open questions of Colin and Ghidaglia [Adv. Differ. Equ. 6 (2001) 1463-1492].
\end{abstract}

Mathematics Subject Classification. 35Q53.

Received March 27, 2012. Revised November 18, 2011.

Published online January 10, 2013.

\section{INTRODUCTION}

In this paper we study a class of Initial-Boundary Value Problems (IBVP) for the Korteweg-de Vries (KdV) equation posed on a finite domain with nonhomogeneous boundary conditions:

$$
\left\{\begin{array}{l}
u_{t}+u_{x}+u_{x x x}+u u_{x}=0, \quad u(x, 0)=\phi(x), \\
u(0, t)=h_{1}(t), u_{x}(L, t)=h_{2}(t), u_{x x}(L, t)=h_{3}(t) .
\end{array} \quad x \in(0, L), t \in \mathbb{R}^{+},\right.
$$

This IBVP can be considered as a model for propagation of surface water waves in the situation where a wavemaker is putting energy in a finite-length channel from the left $(x=0)$ while the right end $(x=L)$ of the channel is free (corresponding the case of $h_{2}=h_{3}=0$ ) (see [16]). The problem was first proposed and studied by Colin and Ghidaglia in the late 1990's [16-18] (cf. also [1-4,8,19,24,25] for other studies of boundary value problems of the KdV equation). In particular, they investigated the well-posedness of the IBVP in the classical Sobolev space $H^{s}(0, L)$ and obtained the following results.

\footnotetext{
Keywords and phrases. The Kortweg-de Vries equation, well-posedness, non-homogeneous boundary value problem.

* The authors would like to thank the referees for their comments and suggestions which have significantly improved the quality of the paper.

** This work was partially supported by a Grant from the Simons Foundation (\#201615 to Bingyu Zhang).

1 Department of Mathematics, Physics, and Computer Science, Raymond Walters College, University of Cincinnati, Cincinnati, 45236 Ohio, USA. eugene.f.kramer@uc.edu

2 Department of Mathematical Sciences, University of Cincinnati, Cincinnati, 45221 Ohio, USA.

rivasie@mail.uc.edu; zhangb@ucmail .uc.edu

3 IMPA, Estrada Dona Castorina 110, 22460-320 Rio de Janeiro, Brasil.
} 
Theorem 1.1 ([18]).

(i) Given $h_{j} \in C^{1}([0, \infty)), j=1,2,3$ and $\phi \in H^{1}(0, L)$ satisfying $h_{1}(0)=\phi(0)$, there exists a $T>0$ such that the IBVP (1.1) admits a solution (in the sense of distribution)

$$
u \in L^{\infty}\left(0, T ; H^{1}(0, L)\right) \cap C\left([0, T] ; L^{2}(0, L)\right) .
$$

(ii) The solution $u$ of the IBVP $[(i i)](1.1)$ exists globally in $H^{1}(0, L)$ if the size of its initial value $\phi \in H^{1}(0, L)$ and its boundary values $h_{j} \in C^{1}([0, \infty)), j=1,2,3$ are all small.

In addition, they showed that the associate linear IBVP

$$
\left\{\begin{array}{l}
u_{t}+u_{x}+u_{x x x}=0, \quad u(x, 0)=\phi(x), \quad x \in(0, L), t \in \mathbb{R}^{+}, \\
u(0, t)=0, u_{x}(L, t)=0, u_{x x}(L, t)=0
\end{array}\right.
$$

possesses the Kato smoothing property: for any $\phi \in L^{2}(0, L)$, the linear IBVP (1.2) admits a unique solution

$$
u \in C\left(\mathbb{R}^{+} ; L^{2}(0, L)\right) \cap L_{l o c}^{2}\left(\mathbb{R}^{+} ; H^{1}(0, L)\right) .
$$

Aided by this smoothing property Colin and Ghidaglia also showed that the homogeneous IBVP (1.1) is locally well-posed in the space $L^{2}(0, L)$.

Theorem 1.2 ([18]). Suppose $h_{1}=h_{2}=h_{3} \equiv 0$, then for any $\phi \in L^{2}(0, L)$, there exists a $T>0$ such that the IBVP (1.1) admits a unique weak solution

$$
u \in C\left([0, T] ; L^{2}(0, L)\right) \cap L^{2}\left(0, T ; H^{1}(0, L)\right) .
$$

In order to encourage further investigation, a series of open problems were proposed by Colin and Ghidaglia [18]. Included in the proposed problems are the following:

\section{Problems:}

(1) Is it possible to prove global existence of solutions of (1.1) for e.g. smooth solutions (as it is the case of the equation posed on a quarter plane or the whole line)?

It is remarked by Colin and Ghidaglia in [18]: "for these problems, uniqueness rely on a priori estimate in $H^{2}$ that we are not able to extend here and therefore establish the existence of more regular solutions".

(2) Is it possible to establish the existence of solutions of (1.1) with their initial value in the space $H^{s}(0, L)$ for some $s<0$ as in the case of the whole line?

Colin and Ghidaglia expected the answer to be positive because of the Kato smoothing property of the associated linear IBVP (1.2).

In this paper, we will continue the work of Colin and Ghidaglia [16-18] by establishing the well-posedness of the IBVP (1.1) in the full strength of Hadamard including existence, uniqueness and continuous dependence and show that the IBVP (1.1) is (locally) well-posed in the space $H^{s}(0, L)$ when $s \geq 0$ and $-\frac{3}{4}<s<0$. In order to describe our results more precisely, let us first introduce some notations.

For given $T>0$ and $s \in \mathbb{R}$, let

$$
\mathbb{H}^{s}(0, T):=H^{\frac{s+1}{3}}(0, T) \times H^{\frac{s}{3}}(0, T) \times H^{\frac{s-1}{3}}(0, T), \quad D_{s, T}:=H^{s}(0, L) \times \mathbb{H}^{s}(0, T)
$$

and

$$
Z_{s, T}=C\left([0, T] ; H^{s}(0, L)\right) \cap L^{2}\left(0, T ; H^{s+1}(0, L)\right) .
$$

For the well-posedness of the IBVP (1.1) that we intend to establish in this paper, some compatibility conditions relating the initial datum $\phi(x)$ and the boundary data $h_{j}(t), j=1,2,3$ are needed. A simple computation 
shows if $u$ is a $C^{\infty}$-smooth solution of the $\operatorname{IBVP}(1.1)$, then its initial data $u(x, 0)=\phi(x)$ and its boundary values $h_{j}(t), j=1,2,3$ must satisfy the following compatibility conditions:

$$
\phi_{k}(0)=h_{1}^{(k)}(0), \quad \phi_{k}^{\prime}(L)=h_{2}^{(k)}(0), \quad \phi_{k}^{\prime \prime}(L)=h_{3}^{(k)}(0)
$$

for $k=0,1, \ldots$, where $h_{j}^{(k)}(t)$ is the $k$-th order derivative of $h_{j}$ and

$$
\left\{\begin{array}{l}
\phi_{0}(x)=\phi(x) \\
\phi_{k}(x)=-\left(\phi_{k-1}^{\prime \prime \prime}(x)+\phi_{k-1}^{\prime}(x)+\frac{1}{2} \sum_{j=0}^{k-1} \frac{(k-1) !}{j !(k-1-j) !}\left(\phi_{j}(x) \phi_{k-j-1}(x)\right)^{\prime}\right)
\end{array}\right.
$$

for $k=1,2, \ldots$ When the well-posedness of (1.1) is considered in the space $H^{s}(0, L)$ for $s \geq 0$, the following $s$-compatibility conditions arise naturally.

Definition 1.3 (s-compatibility). Let $T>0$ and $s \geq 0$ be given. A four-tuple

$$
(\phi, \boldsymbol{h})=\left(\phi, h_{1}, h_{2}, h_{3}\right) \in D_{s, T}
$$

is said to be $s$-compatible with respect to the IBVP (1.1) if

$$
\phi_{k}(0)=h_{1}^{(k)}(0)
$$

when $k=0,1, \ldots[s / 3]$ and $\frac{1}{2}<s-3[s / 3] \leq 3 / 2$,

$$
\phi_{k}(0)=h_{1}^{(k)}(0), \quad \phi_{k}^{\prime}(L)=h_{2}^{(k)}(0)
$$

when $k=0,1, \ldots[s / 3]$ and $\frac{3}{2}<s-3[s / 3] \leq 5 / 2$ and

$$
\phi_{k}(0)=h_{1}^{(k)}(0), \quad \phi_{k}^{\prime}(L)=h_{2}^{(k)}(0) \quad \phi_{k}^{\prime \prime}(L)=h_{3}^{(k)}(0)
$$

when $k=0,1, \ldots[s / 3]$ and

$$
5 / 2<s-3[s / 3] \leq 3,
$$

or when $k=0,1, \ldots[s / 3]-1$ and

$$
0 \leq s-3[s / 3] \leq \frac{1}{2}
$$

The following theorem regarding the local well-posedness of the IBVP $(1.1)$ in the space $H^{s}(0, L)$ for any $s \geq 0$, is one of the main results of this paper.

Theorem 1.4. Let $s \geq 0, T>0$ and $r>0$ be given with

$$
s \neq \frac{2 j-1}{2}, \quad j=1,2, \ldots
$$

There exists a $T^{*} \in(0, T]$ such that for any s-compatible

$$
(\phi, \boldsymbol{h}) \in D_{s, T}
$$

with 4

the IBVP (1.1) admits a unique solution

$$
\|(\phi, h)\|_{D_{0, T}} \leq r
$$

$$
u \in C\left(\left[0, T^{*}\right] ; H^{s}(0, L)\right) \cap L^{2}\left(0, T^{*} ; H^{s+1}(0, L)\right) .
$$

Moreover, the corresponding solution map is Lipschitz continuous ${ }^{5}$.

\footnotetext{
${ }^{4}$ It is worth to point out here that it is not required that $\|(\phi, h)\|_{D_{s, T}} \leq r$ when $s>0$.

${ }^{5}$ The solution map, is in fact, real analytic (cf. [49-51]).
} 
To consider the well-posedness of the IBVP $(1.1)$ in the space $H^{s}(0, L)$ with $s<0$, the following Bourgain-type spaces will be used (cf. $[7,11,20,26-30,32])$.

For any given $s \in \mathbb{R}, 0 \leq b \leq 1,0 \leq \alpha \leq 1$ and function $w \equiv w(x, t): \mathbb{R}^{2} \rightarrow \mathbb{R}$, define

$$
\begin{aligned}
& \Lambda_{s, b}(w)=\left(\int_{-\infty}^{\infty} \int_{-\infty}^{\infty}\left\langle\tau-\left(\xi^{3}-\xi\right)\right\rangle^{2 b}\langle\xi\rangle^{2 s}|\hat{w}(\xi, \tau)|^{2} \mathrm{~d} \xi \mathrm{d} \tau\right)^{\frac{1}{2}}, \\
& \lambda_{\alpha}(w)=\left(\int_{-\infty}^{\infty} \int_{|\xi| \leq 1}\langle\tau\rangle^{2 \alpha}|\hat{w}(\xi, \tau)|^{2} \mathrm{~d} \xi \mathrm{d} \tau\right)^{\frac{1}{2}}
\end{aligned}
$$

and

$$
\mathcal{G}_{s}(w)=\left(\int_{-\infty}^{\infty}\langle\xi\rangle^{2 s}\left(\int_{-\infty}^{\infty} \frac{|\hat{w}(\xi, \tau)|}{1+\left|\tau-\left(\xi^{3}-\xi\right)\right|} \mathrm{d} \tau\right)^{2} \mathrm{~d} \xi\right)^{1 / 2}
$$

where $\langle\cdot\rangle:=\left(1+|\cdot|^{2}\right)^{\frac{1}{2}}$. Let $X_{s, b}$ be the space of all functions $w$ satisfying

$$
\|w\|_{X_{s, b}}:=\Lambda_{s, b}(w)<\infty
$$

while $Y_{s, b}$ be the space of all functions $w$ satisfying

$$
\|w\|_{Y_{s, b}}:=\left(\mathcal{G}_{s}^{2}(w)+\Lambda_{s,-b}^{2}(w)\right)^{1 / 2}<\infty .
$$

In addition, let $X_{s, b}^{\alpha}$ be the space of all functions $w$ satisfying

$$
\|w\|_{X_{s, b}^{\alpha}}:=\left(\Lambda_{s, b}^{2}(w)+\lambda_{\alpha}^{2}(w)\right)^{1 / 2}<\infty
$$

and let $Y_{s, b}^{\alpha}$ be the space of all $w$ satisfying

$$
\|w\|_{Y_{s, b}^{\alpha}}:=\left(\lambda_{\alpha-1}^{2}(w)+\mathcal{G}_{s}^{2}(w)+\Lambda_{s,-b}^{2}(w)\right)^{1 / 2}<\infty .
$$

The spaces $X_{s, b}, Y_{s, b}, X_{s, b}^{\alpha}$ and $Y_{s, b}^{\alpha}$ are all Banach spaces. $X_{s, b}$ and $X_{s, b}^{\alpha}$ are equivalent when $b \geq \alpha$. The spaces $Y_{s, b}$ and $X_{s,-b}$ are also equivalent when $b<\frac{1}{2}$. Furthermore, let

$$
\mathcal{X}_{s, b}^{\alpha} \equiv C_{b}\left(\mathbb{R} ; H^{s}(\mathbb{R})\right) \cap X_{s, b}^{\alpha}
$$

with the norm

$$
\|w\|_{\mathcal{X}_{s, b}^{\alpha}}=\left(\sup _{t \in \mathbb{R}}\|w(\cdot, t)\|_{H^{s}(\mathbb{R})}^{2}+\|w\|_{X_{s, b}^{\alpha}}^{2}\right)^{1 / 2}
$$

The Bourgain-type spaces given above are defined for functions posed on the whole plane $\mathbb{R} \times \mathbb{R}$. However the IBVP $(1.1)$ is posed on the finite domain $(0, L) \times(0, T)$. It is thus natural to introduce a restricted version of the Bourgain space $X_{s, b}$ to the domain $(0, L) \times(0, T)$ as follows:

$$
X_{s, b}^{T}=\left.X_{s, b}\right|_{(0, L) \times(0, T)}
$$

with the quotient norm

$$
\|u\|_{X_{s, b}^{T}} \equiv \inf _{w \in X_{s, b}}\left\{\|w\|_{X_{s, b}}: w(x, t)=u(x, t) \text { on }(0, L) \times(0, T)\right\}
$$

for any $u(x, t)$ defined on $(0, L) \times(0, T)$. The spaces $Y_{s, b}^{T}, X_{s, b}^{\alpha, T}, Y_{s, b}^{\alpha, T}$ and $\mathcal{X}_{s, b}^{\alpha, T}$ are defined similarly.

Another main result of this paper addresses the second problem listed previously from Colin and Ghidaglia [18]. The research presented establishes the local well-posedness of the IBVP (1.1) in the space $H^{s}(0, L)$ for some $s<0$. 
Theorem 1.5. Let $s \in\left(-\frac{3}{4}, 0\right), T>0$ and $r>0$ be given. There exist $T^{*} \in(0, T]$ and $\alpha>\frac{1}{2}$ such that for any

$$
(\phi, \boldsymbol{h}) \in D_{s, T}
$$

satisfying

$$
\|(\phi, \boldsymbol{h})\|_{D_{s, T}} \leq r
$$

the IBVP (1.1) admits a unique solution $u \in C\left(\left[0, T^{*}\right] ; H^{s}(0, L)\right)$ with

$$
\|u\|_{\mathcal{X}_{s, \frac{1}{2}}^{\alpha, T^{*}}}<+\infty
$$

Moreover, the corresponding solution map is Lipschitz continuous.

The following remarks are in order.

(i) Theorem 1.4 states that the IBVP (1.1) is well-posed in the space $H^{s}(0, L)$ for any $s \geq 0$, not just for $s=0$ or $s=1$. In particular, it demonstrates the existence of classical solutions and shows that the smoother the initial value and boundary data, the smoother the corresponding solution.

(ii) To have solution $u$ in the space $C\left([0, T] ; H^{s}(0, L)\right)$, Theorem 1.4 only requires that the initial value $\phi \in$ $H^{s}(0, L)$ and the boundary data be

$$
h_{1} \in H^{\frac{s+1}{3}}(0, T), \quad h_{2} \in H^{\frac{s}{3}}(0, T), \quad h_{3} \in H^{\frac{s-1}{3}}(0, T) .
$$

In particular, if $s=1$, it is sufficient to require that

$$
h_{1} \in H^{\frac{2}{3}}(0, T), \quad h_{2} \in H^{\frac{1}{3}}(0, T), \quad h_{3} \in L^{2}(0, T),
$$

rather than $h_{j} \in C^{1}(0, T), j=1,2,3$ as in Theorem 1.1. Moreover, condition (1.10) is optimal in order to have the corresponding solution $u \in C\left([0, T] ; H^{s}(0, L)\right)$.

(iii) In Theorem 1.4, the life span $\left(0, T^{*}\right)$ of the solutions depends only $\|(\phi, \boldsymbol{h})\|_{D_{0, T}}$, not on $\|(\phi, \boldsymbol{h})\|_{D_{s, T}}$ when $s>0$, in particular. Thus a solution $u$ blows up in the space $H^{s}(0, L)$ for any $s>0$ if and only if it blows up in the space $L^{2}(0, L)$.

(iv) Taking lead from the recent works of Bona et al. [9], Molinet [35], and Molinet and Vento [37], we conjecture that the IBVP $(1.1)$ is locally well-posed in the space $H^{s}(0, L)$ for $-1<s \leq-\frac{3}{4}$, but is ill-posed in the space $H^{s}(0, L)$ for any $s<-1$.

It is also interesting and constructive to compare the study of the IBVP (1.1) with another class of IBVP of the $\mathrm{KdV}$ equation posed on the finite domain $(0, L)$. The following problem has been well studied over the past few years $[5,9,26,48]$.

$$
\left\{\begin{array}{l}
u_{t}+u_{x}+u_{x x x}+u u_{x}=0, \quad x \in(0, L), t \in \mathbb{R}^{+}, \\
u(x, 0)=\phi(x), \\
u(0, t)=h_{1}(t), u(L, x)=h_{2}(t), u_{x}(L, t)=h_{3}(t) .
\end{array}\right.
$$

While the study of the IBVP (1.11) goes back as early as late 1970s [12,13], the nonhomogeneous IBVP (1.11) was first shown by Bona et al. [5] to be locally well-posed in the space $H^{s}(0, L)$ for any $s \geq 0$ :

Let $s \geq 0, r>0$ and $T>0$ be given. There exists $T^{*} \in(0, T]$ such that for any s-compatible

$$
\phi \in H^{s}(0, L), \quad \boldsymbol{h}=\left(h_{1}, h_{2}, h_{3}\right) \in H^{\frac{s+1}{3}}(0, T) \times H^{\frac{s+1}{3}}(0, T) \times H^{\frac{s}{3}}(0, T)
$$

\footnotetext{
${ }^{6}$ See [5] for the exact definition of $s$-compatibility for the IBVP (1.11).
} 
satisfying ${ }^{7}$

$$
\|\phi\|_{H^{s}(0, L)}+\|\boldsymbol{h}\|_{H^{\frac{s+1}{3}}(0, T) \times H^{\frac{s+1}{3}}(0, T) \times H^{\frac{s}{3}}(0, T)} \leq r
$$

the IBVP (1.11) admits a unique solution

$$
u \in C\left(\left[0, T^{*}\right] ; H^{s}(0, L)\right) \cap L^{2}\left(0, T^{*} ; H^{s+1}(0, L)\right) .
$$

Moreover, the corresponding solution map is Lipschitz continuous in the corresponding spaces.

Later Holmer [26] showed that the IBVP (1.11) is locally well-posed in the space $H^{s}(0, L)$ for any $-\frac{3}{4}<s<\frac{1}{2}$ :

Let $s \in\left(-\frac{3}{4}, \frac{1}{2}\right), r>0$ and $T>0$ be given. There exists a $T^{*} \in(0, T]$ such that for any

$$
\phi \in H^{s}(0, L), \quad \boldsymbol{h}=\left(h_{1}, h_{2}, h_{3}\right) \in H^{\frac{s+1}{3}}(0, T) \times H^{\frac{s+1}{3}}(0, T) \times H^{\frac{s}{3}}(0, T)
$$

satisfying

$$
\|\phi\|_{H^{s}(0, L)}+\|\boldsymbol{h}\|_{H^{\frac{s+1}{3}}(0, T) \times H^{\frac{s+1}{3}}(0, T) \times H^{\frac{s}{3}}(0, T)} \leq r,
$$

the IBVP (1.11) admits a unique mild solution ${ }^{8}$

$$
u \in C\left(\left[0, T^{*}\right] ; H^{s}(0, L)\right) .
$$

Moreover, the corresponding solution map is Lipschitz continuous in the corresponding spaces.

${ }^{7}$ This condition can be weakened as

$$
\|\phi\|_{L^{2}(0, L)}+\|\boldsymbol{h}\|_{H^{\frac{1}{3}}(0, T) \times H^{\frac{1}{3}}(0, T) \times L^{2}(0, T)} \leq r .
$$

${ }^{8} \mathrm{~A}$ function $u \in C\left(\left[0, T^{*}\right] ; H^{s}(0, L)\right)$ is said to be a mild solution of the IBVP (1.11) if there exist a sequence

$$
u_{n} \in C^{1}\left(\left[0, T^{*}\right] ; L^{2}(0, L)\right) \cap C\left[\left(0, T^{*}\right] ; H^{3}(0, L)\right), \quad n=1,2, \ldots
$$

solving the equation in (1.11) and as $n \rightarrow \infty$,

$$
\begin{gathered}
u_{n} \rightarrow u \quad \text { in } C\left(\left[0, T^{*}\right] ; H^{s}(0, L)\right), \\
h_{1, n}:=u_{n}(0, \cdot) \rightarrow h_{1}, \quad h_{2, n}:=u(L, \cdot) \rightarrow h_{2} \quad \text { in } H^{\frac{s+1}{3}}\left(0, T^{*}\right)
\end{gathered}
$$

and

$$
h_{3, n}:=\partial_{x} u_{n}(L, \cdot) \rightarrow h_{3} \quad \text { in } H^{\frac{s}{3}}\left(0, T^{*}\right)
$$


More recently, Bona et al. [9] showed that the IBVP (1.11) is locally well-posed $H^{s}(0, L)$ for any $-1<s \leq 0$.

Let $r>0,-1<s \leq 0$ and $T>0$ be given. There exists a $T^{*} \in(0, T]$ such that for any

$$
\phi \in H^{s}(0, L), \quad \boldsymbol{h}=\left(h_{1}, h_{2}, h_{3}\right) \in H^{\frac{s+1}{3}}(0, T) \times H^{\frac{s+1}{3}}(0, T) \times H^{\frac{s}{3}}(0, T)
$$

satisfying

$$
\|\phi\|_{H^{s}(0, L)}+\|\boldsymbol{h}\|_{H^{\frac{s+1}{3}}(0, T) \times H^{\frac{s+1}{3}}(0, T) \times H^{\frac{s}{3}}(0, T)} \leq r,
$$

the IBVP (1.11) admits a unique mild solution

$$
u \in C\left(\left[0, T^{*}\right] ; H^{s}(0, L)\right) .
$$

Moreover, the corresponding solution map is Lipschitz continuous in the corresponding spaces.

Although there is only a slight difference between the boundary conditions of IBVP (1.1) and the IBVP (1.11), there is a big gap between their well-posedness results. For the IBVP (1.1), the well-posedness results presented in Theorems 1.4 and 1.5 are local in the sense that the time interval $\left(0, T^{*}\right)$ in which the solution exists depends on $r$ and, in general, the larger the $r$, the smaller the $T^{*}$. By contrast, the IBVP (1.11) is known to be globally well-posed in the space $H^{s}(0, L)$ for any $s \geq 0$ in the sense that one always has $\left(0, T^{*}\right)=(0, T)$ no matter how large the value of $r$ is $(c f .[5,23])$. This difference stems from the fact that the $L^{2}$-energy of the solution of the homogeneous IBVP $(1.11)(\boldsymbol{h}=0)$ is decreasing since

$$
\frac{\mathrm{d}}{\mathrm{d} t} \int_{0}^{L} u^{2}(x, t) \mathrm{d} x=-u_{x}^{2}(0, t) \quad \text { for } \quad t \geq 0,
$$

while for the homogeneous IBVP (1.1) it is not clear, in general, whether the $L^{2}$-energy of its solution is increasing or decreasing since

$$
\frac{\mathrm{d}}{\mathrm{d} t} \int_{0}^{L} u^{2}(x, t)=-u^{2}(L, t)-\frac{2}{3} u^{3}(L, t)-u_{x}^{2}(0, t) \quad \text { for } \quad t \geq 0 .
$$

The approach used in the proofs of their results in $[5,22,26]$ is very much different from what used in the proof of Theorem 1.1, but more or less along the line used in the proof of Theorem 1.2, in which the Kato smoothing property of the associated linear system plays an important role. In this paper, we will use a similar approach as that developed in $[5,7]$ but with some modifications to prove Theorems 1.4 and 1.5. The key ingredients of the approach are listed below.

(1) An explicit solution formula will be derived for the following nonhomogeneous boundary value problem of the linear equation,

$$
\left\{\begin{array}{l}
v_{t}+v_{x}+v_{x x x}=0, \quad x \in(0, L), \quad t \geq 0 \\
v(x, 0)=0 \\
v(0, t)=h_{1}(t), v_{x}(L, t)=h_{2}(t), v_{x x}(L, t)=h_{3}(t),
\end{array}\right.
$$

which not only enables us to establish the well-posedness of the IBVP (1.1) with the optimal regularity conditions imposed on the boundary data, but also plays an important role in obtaining the well-posedness of the IBVP (1.1) in the space $H^{s}(0, L)$ with $-\frac{3}{4}<s<0$.

(2) The Kato smoothing property of the associated linear problem

$$
\left\{\begin{array}{l}
v_{t}+v_{x}+v_{x x x}=f, \quad x \in(0, L), \quad t \geq 0 \\
v(x, 0)=\phi(x), \\
v(0, t)=h_{1}(t), v_{x}(L, t)=h_{2}(t), v_{x x}(L, t)=h_{3}(t) .
\end{array}\right.
$$


For given $T>0$, there exists a constant $C>0$ such that the solution $v$ of (1.13) satisfies

$$
\|v\|_{Z_{0, T}} \leq C\left(\|(\phi, \boldsymbol{h})\|_{D_{0, T}}+\|f\|_{L^{1}\left(0, T ; L^{2}(0, L)\right)}\right)
$$

for any $(\phi, \boldsymbol{h}) \in D_{0, T}$ and $f \in L^{1}\left(0, T ; L^{2}(0, L)\right)$. This property is an extension of the Kato smoothing property obtained by Colin and Ghidaglia [18] for the nonhomogeneous problem.

(3) Tartar's nonlinear interpolation theorem [47].

Let $B_{0}$ and $B_{1}$ be two Banach spaces such that $B_{1} \subset B_{0}$ with continuous inclusion map. Let $f \in B_{0}$ and, for $t \geq 0$, define

$$
K(f, t)=\inf _{g \in B_{1}}\left\{\|f-g\|_{B_{0}}+t\|g\|_{B_{1}}\right\} .
$$

For $0 \leq \theta \leq 1$, the (real) interpolation of $B_{0}$ and $B_{1}$ is defined by

$$
\left[B_{0}, B_{1}\right]_{\theta}=\left\{f \in B_{0}:\|f\|_{B_{\theta}}:=\left(\int_{0}^{\infty} K^{2}(f, t) t^{-2 \theta-1} \mathrm{~d} t\right)^{\frac{1}{2}}<+\infty\right\} .
$$

Then $B_{\theta}$ is a Banach space with norm $\|\cdot\|_{B_{\theta}}$.

Theorem 1.6 (Tartar [47]). For $j=1,2$, let $B_{0}^{j}$ and $B_{1}^{j}$ be Banach spaces such that $B_{1}^{j} \subset B_{0}^{j}$ with continuous inclusion mappings. Suppose $A$ is a mapping such that

(i) $A: B_{0}^{1} \rightarrow B_{0}^{2}$ and for $f, g \in B_{0}^{1}$,

$$
\|A f-A g\|_{B_{0}^{2}} \leq C_{0}\left(\|f\|_{B_{0}^{1}}+\|g\|_{B_{0}^{1}}\right)\|f-g\|_{B_{0}^{1}}
$$

and

(ii) $A: B_{1}^{1} \rightarrow B_{1}^{2}$ and for $h \in B_{1}^{1}$

$$
\|A h\|_{B_{1}^{2}} \leq C_{1}\left(\|h\|_{B_{0}^{1}}\right)\|h\|_{B_{1}^{1}}
$$

where $C_{j}: \mathbb{R}^{+} \rightarrow \mathbb{R}^{+}$are continuous non-decreasing functions, $j=0,1$.

Then if $0 \leq \theta \leq 1, A$ maps $B_{\theta}^{1}$ into $B_{\theta}^{2}$ and for $f \in B_{\theta}^{1}$,

$$
\|A f\|_{B_{\theta}^{2}} \leq C\left(\|f\|_{B_{0}^{1}}\right)\|f\|_{B_{\theta}^{1}}
$$

where for $r>0, C(r)=4 C_{0}(4 r)^{1-\theta} C_{1}(3 r)^{\theta}$.

Note that if $B_{0}^{1}=D_{0, T}, B_{1}^{1}=D_{3, T}, B_{1}^{1}=Z_{0, T}$ and $B_{1}^{2}=Z_{3, T}$. Then

$$
D_{s, T}=\left[B_{0}^{1}, B_{1}^{1}\right]_{\theta}, \quad Z_{s, T}=\left[B_{0}^{2}, B_{1}^{2}\right]_{\theta}
$$

with $\theta=\frac{s}{3}, 0 \leq s \leq 3$. With the help of this theorem, we will only need to consider the case of $s=3 k, k=$ $0,1,2, \ldots$ when proving Theorem 1.4 .

(4) Following Bona et al. [7], the IBVP (1.1) will be converted to an integral equation posed on the whole line $\mathbb{R}$ which makes it possible to conduct Bourgain spaces analysis to obtain the well-posedness of the IBVP (1.1) in $H^{s}(0, L)$ for $-\frac{3}{4}<s<0$.

This paper is organized as follows. In Section 2, the various linear problems associated to the IBVP (1.1) are studied. Section 3 is devoted to the well-posedness of the nonlinear IBVP (1.1). Concluding remarks and open questions for further investigations are presented in Section 4. 


\section{LINEAR PROBLEMS}

\subsection{The boundary integral operators}

Consider the nonhomogeneous boundary-value problem

$$
\left\{\begin{array}{l}
v_{t}+v_{x}+v_{x x x}=0, \quad v(x, 0)=0, \quad x \in(0, L), \quad t \geq 0 \\
v(0, t)=h_{1}(t), v_{x}(L, t)=h_{2}(t), \quad v_{x x}(L, t)=h_{3}(t)
\end{array}\right.
$$

We look for an explicit solution formula for the IBVP (2.1). Without loss of generality, we assume that $L=1$ in this subsection. Applying the Laplace transform yo both sides of the equation in (2.1) with respect to $t$, the IBVP (2.1) is converted to

$$
\left\{\begin{array}{l}
s \hat{v}+\hat{v}_{x}+\hat{v}_{x x x}=0, \\
\hat{v}(0, s)=\hat{h}_{1}(s), \hat{v}_{x}(1, s)=\hat{h}_{2}(s), \hat{v}_{x x}(1, s)=\hat{h}_{3}(s),
\end{array}\right.
$$

where

$$
\hat{v}(x, s)=\int_{0}^{+\infty} \mathrm{e}^{-s t} v(x, t) \mathrm{d} t
$$

and

$$
\hat{h}_{j}(s)=\int_{0}^{\infty} \mathrm{e}^{-s t} h_{j}(t) \mathrm{d} t, \quad j=1,2,3 .
$$

The solution of (2.2) can be written in the form

$$
\hat{v}(x, s)=\sum_{j=1}^{3} c_{j}(s) \mathrm{e}^{\lambda_{j}(s) x}
$$

where $\lambda_{j}(s), j=1,2,3$ are solutions of the characteristic equation

$$
s+\lambda+\lambda^{3}=0
$$

and $c_{j}(s), j=1,2,3$, solve the linear system

$$
\underbrace{\left(\begin{array}{ccc}
1 & 1 & 1 \\
\lambda_{1} \mathrm{e}^{\lambda_{1}} & \lambda_{2} \mathrm{e}^{\lambda_{2}} & \lambda_{3} \mathrm{e}^{\lambda_{3}} \\
\lambda_{1}^{2} \mathrm{e}^{\lambda_{1}} & \lambda_{2}^{2} \mathrm{e}^{\lambda_{2}} & \lambda_{3}^{2} \mathrm{e}^{\lambda_{3}}
\end{array}\right)}_{A}\left(\begin{array}{c}
c_{1} \\
c_{2} \\
c_{3}
\end{array}\right)=\underbrace{\left(\begin{array}{l}
\hat{h}_{1} \\
\hat{h}_{2} \\
\hat{h}_{3}
\end{array}\right)}_{\hat{h}} .
$$

By Cramer's rule,

$$
c_{j}=\frac{\Delta_{j}(s)}{\Delta(s)}, j=1,2,3
$$

where $\Delta$ is the determinant of $A$ and $\Delta_{j}$ is the determinant of the matrix $A$ with the column $j$ replaced by $\widehat{\boldsymbol{h}}$. Taking the inverse Laplace transform of $\widehat{v}$ and following similar arguments as those in [5] yield the representation

$$
v(x, t)=\sum_{m=1}^{3} v_{m}(x, t)
$$

with

$$
v_{m}(x, t)=\sum_{j=1}^{3} v_{j, m}(x, t)
$$


and

$$
v_{j, m}(x, t)=v_{j, m}^{+}(x, t)+v_{j, m}^{-}(x, t),
$$

where

$$
v_{j, m}^{+}(x, t)=\frac{1}{2 \pi i} \int_{0}^{+i \infty} \mathrm{e}^{s t} \frac{\Delta_{j, m}(s)}{\Delta(s)} \hat{h}_{m}(s) \mathrm{e}^{\lambda_{j}(s) x} \mathrm{~d} s
$$

and

$$
v_{j, m}^{-}(x, t)=\frac{1}{2 \pi i} \int_{-i \infty}^{0} \mathrm{e}^{s t} \frac{\Delta_{j, m}(s)}{\Delta(s)} \hat{h}_{m}(s) \mathrm{e}^{\lambda_{j}(s) x} \mathrm{~d} s
$$

for $j, m=1,2,3$. Here $\Delta_{j, m}(s)$ is obtained from $\Delta_{j}(s)$ by letting $\hat{h}_{m}(s)=1$ and $\hat{h}_{k}(s)=0$ for $k \neq m$, $k, m=1,2,3$. Making the substitution $s=i\left(\rho^{3}-\rho\right)$ with $1<\rho<\infty$ in the characteristic equation

$$
s+\lambda+\lambda^{3}=0
$$

gives the following roots in terms of $\rho$

$$
\lambda_{1}^{+}(\rho)=i \rho, \quad \lambda_{2}^{+}(\rho)=\frac{\sqrt{3 \rho^{2}-4}-i \rho}{2} \quad \lambda_{3}^{+}(\rho)=\frac{-\sqrt{3 \rho^{2}-4}-i \rho}{2} .
$$

Thus $v_{j, m}^{+}(x, t)$ has the form

$$
v_{j, m}^{+}(x, t)=\frac{1}{2 \pi} \int_{1}^{\infty} \mathrm{e}^{\mathrm{i}\left(\rho^{3}-\rho\right) t} \frac{\Delta_{j, m}^{+}(\rho)}{\Delta^{+}(\rho)} \hat{h}_{m}^{+}(\rho) \mathrm{e}^{\lambda_{j}^{+}(\rho) x}\left(3 \rho^{2}-1\right) \mathrm{d} \rho
$$

and

$$
v_{j, m}^{-}(x, t)=\overline{v_{j, m}^{+}(x, t)}
$$

where $\hat{h}_{m}^{+}(\rho)=\hat{h}_{m}\left(i\left(\rho^{3}-\rho\right)\right), \Delta^{+}(\rho)$ and $\Delta_{j, m}^{+}(\rho)$ are obtained from $\Delta(s)$ and $\Delta_{j, m}(s)$ by replacing $s$ with $i\left(\rho^{3}-\rho\right)$ and $\lambda_{j}(s)$ by $\lambda_{j}^{+}(\rho)=\lambda_{j}\left(i\left(\rho^{3}-\rho\right)\right)$.

For given $m, j=1,2,3$, let $W_{j, m}$ be an operator on $H_{0}^{s}\left(\mathbb{R}^{+}\right)$defined as follows:

$$
\left[W_{j, m} h\right](x, t) \equiv\left[U_{j, m} h\right](x, t)+\overline{\left[U_{j, m} h\right](x, t)}
$$

for any $h \in H_{0}^{s}\left(\mathbb{R}^{+}\right)$with

$$
\begin{aligned}
{\left[U_{j, m} h\right](x, t) } & \equiv \frac{1}{2 \pi} \int_{1}^{+\infty} \mathrm{e}^{\mathrm{i}\left(\rho^{3}-\rho\right) t} \mathrm{e}^{-\lambda_{j}^{+}(\rho)(1-x)}\left(3 \rho^{2}-1\right)\left[Q_{j, m}^{+} h\right](\rho) \mathrm{d} \rho \\
& =\frac{1}{2 \pi} \int_{1}^{+\infty} \mathrm{e}^{\mathrm{i}\left(\rho^{3}-\rho\right) t} \mathrm{e}^{-\lambda_{j}^{+}(\rho) x^{\prime}}\left(3 \rho^{2}-1\right)\left[Q_{j, m}^{+} h\right](\rho) \mathrm{d} \rho, \quad\left(x^{\prime}=1-x\right),
\end{aligned}
$$

for $j=1,2, m=1,2,3$ and

$$
\left[U_{3, m} h\right](x, t) \equiv \frac{1}{2 \pi} \int_{1}^{+\infty} \mathrm{e}^{\mathrm{i}\left(\rho^{3}-\rho\right) t} \mathrm{e}^{\lambda_{3}^{+}(\rho) x}\left(3 \rho^{2}-1\right)\left[Q_{3, m}^{+} h\right](\rho) \mathrm{d} \rho
$$

for $m=1,2,3$, where

$$
\left[Q_{3, m}^{+} h\right](\rho):=\frac{\Delta_{3, m}^{+}(\rho)}{\Delta^{+}(\rho)} \hat{h}^{+}(\rho), \quad\left[Q_{j, m}^{+} h\right](\rho)=\frac{\Delta_{j, m}^{+}(\rho)}{\Delta^{+}(\rho)} \mathrm{e}^{\lambda_{j}^{+}(\rho)} \hat{h}^{+}(\rho)
$$

for $j=1,2$ and $m=1,2,3, \hat{h}^{+}(\rho)=\hat{h}\left(i\left(\rho^{3}-\rho\right)\right)$. The solution of the IBVP (2.1) then has the following representation. 
Lemma 2.1. Given $h_{1}, h_{2}$ and $h_{3}$, defining $\boldsymbol{h}=\left(h_{1}, h_{2}, h_{3}\right)$. The solution $v$ of the IBVP (2.1) can be written in the form

$$
v(x, t)=\left[W_{b d r} \boldsymbol{h}\right](x, t):=\sum_{j, m=1}^{3}\left[W_{j, m} h_{m}\right](x, t) .
$$

\subsection{Linear estimates}

Consider the IBVP of the linear equation:

$$
\left\{\begin{array}{l}
v_{t}+v_{x}+v_{x x x}=f, \quad x \in(0, L), \quad t \geq 0 \\
v(x, 0)=\phi(x) \\
v(0, t)=0, v_{x}(L, t)=0, v_{x x}(L, t)=0
\end{array}\right.
$$

By standard semigroup theory [39], for any $\phi \in L^{2}(0, L)$ and $f \in L^{1}\left(0, T ; L^{2}(0, L)\right),(2.9)$ admits a unique solution $v \in C\left([0, T] ; L^{2}(0, L)\right)$, which can be written in the form

$$
v(t)=W_{0}(t) \phi+\int_{0}^{t} W_{0}(t-\tau) f(\tau) \mathrm{d} \tau
$$

where $W_{0}$ is the $C_{0}$-semigroup in the space $L^{2}(0, L)$ generated by the linear operator

$$
A \psi=-\psi^{\prime \prime \prime}-\psi^{\prime}
$$

with the domain

$$
\mathcal{D}(A)=\left\{\psi \in H^{3}(0, L): \psi(0)=\psi^{\prime}(L)=\psi^{\prime \prime}(L)=0\right\} .
$$

Proposition 2.2. Let $T>0$ be given. There exists a constant $C$ such that for any $\phi \in L^{2}(0, L)$ and $f \in$ $L^{1}\left(0, T ; L^{2}(0, L)\right)$, the corresponding solution $v$ of the IBVP $(2.9)$ belongs to the space $Z_{0, T}$ and

$$
\|v\|_{Z_{0, T}} \leq C\left(\|\phi\|+\|f\|_{L^{1}\left(0, T ; L^{2}(0, L)\right)}\right) .
$$

Proof. First multiplying the both sides of the equation in $(2.9)$ by $2 v$ and integrating over $(0, L)$ with respect to $x$ gives

$$
\frac{\mathrm{d}}{\mathrm{d} t} \int_{0}^{L} v^{2}(x, t) \mathrm{d} x+v^{2}(L, t)+v_{x}^{2}(0, t)=2 \int_{0}^{L} x f(x, t) v(x, t) \mathrm{d} x .
$$

Thus

$$
\sup _{0<t<T}\|v(\cdot, t)\|_{L^{2}(0, L)} \leq\|\phi\|_{L^{2}(0, L)}+C\|f\|_{L^{1}\left(0, T ; L^{2}(0, L)\right)} .
$$

Then multiplying the both sides of the equation in $(2.9)$ by $2 x v$ and integrating over $(0, L)$ with respect to $x$ yields

$$
\frac{\mathrm{d}}{\mathrm{d} t} \int_{0}^{L} x v^{2}(x, t) \mathrm{d} x+L v^{2}(L, t)+3 \int_{0}^{L} v_{x}^{2} \mathrm{~d} x=\int_{0}^{L} v^{2} \mathrm{~d} x+2 \int_{0}^{L} f(x, t) v(x, t) \mathrm{d} x .
$$

Estimate (2.10) then follows easily.

Next we consider the nonhomogeneous boundary-value problem

$$
\left\{\begin{array}{l}
v_{t}+v_{x}+v_{x x x}=0, \quad x \in(0, L), \quad t \geq 0, \\
v(x, 0)=0, \\
v(0, t)=h_{1}(t), v_{x}(L, t)=h_{2}(t), v_{x x}(L, t)=h_{3}(t) .
\end{array}\right.
$$

We have the following estimate for its solutions. 
Proposition 2.3. For given $T>0$, there exists a constant $C$ such that for any $\boldsymbol{h} \in \mathbb{H}^{0}(0, T)$, the corresponding solution $v$ of the system (2.11) belongs to the space $Z_{0, T}$ and

$$
\|v\|_{Z_{0, T}} \leq C\|\boldsymbol{h}\|_{\mathbb{H}^{0}(0, T)} .
$$

Proof. From Section 2.1 we have

$$
\lambda_{1}^{+}(\rho)=i \rho, \quad \lambda_{2}^{+}(\rho)=\frac{\sqrt{3 \rho^{2}-4}-i \rho}{2} \quad \lambda_{3}^{+}(\rho)=\frac{-\sqrt{3 \rho^{2}-4}-i \rho}{2} .
$$

The asymptotic behaviors of the ratios $\frac{\Delta_{j, m}^{+}(\rho)}{\Delta^{+}(\rho)}$ for $\rho \rightarrow+\infty$ are listed below.

\begin{tabular}{|c|c|c|}
\hline$\frac{\Delta_{1,1}^{+}(\rho)}{\Delta^{+}(\rho)} \sim \mathrm{e}^{-\frac{\sqrt{3}}{2} \rho}$ & $\frac{\Delta_{2,1}^{+}(\rho)}{\Delta^{+}(\rho)} \sim \mathrm{e}^{-\sqrt{3} \rho}$ & $\frac{\Delta_{3,1}^{+}(\rho)}{\Delta^{+}(\rho)} \sim 1$ \\
\hline$\frac{\Delta_{1,2}^{+}(\rho)}{\Delta^{+}(\rho)} \sim \rho^{-1}$ & $\frac{\Delta_{2,2}^{+}(\rho)}{\Delta^{+}(\rho)} \sim \rho^{-1} \mathrm{e}^{-\frac{\sqrt{3}}{2} \rho}$ & $\frac{\Delta_{3,2}^{+}(\rho)}{\Delta^{+}(\rho)} \sim \rho^{-1}$ \\
\hline$\frac{\Delta_{1,3}^{+}(\rho)}{\Delta^{+}(\rho)} \sim \rho^{-2}$ & $\frac{\Delta_{2,3}^{+}(\rho)}{\Delta^{+}(\rho)} \sim \rho^{-2} \mathrm{e}^{-\frac{\sqrt{3}}{2} \rho}$ & $\frac{\Delta_{3,3}^{+}(\rho)}{\Delta^{+}(\rho)} \sim \rho^{-2}$ \\
\hline
\end{tabular}

For $m=1,2,3$ and $j=1,2$, set

$$
{\hat{h^{*}}}_{3, m}^{+}(\rho):=\left[Q_{3, m}^{+} h_{m}\right](\rho)=\frac{\Delta_{3, m}^{+}(\rho)}{\Delta^{+}(\rho)} \hat{h}_{m}^{+}(\rho)
$$

and

$$
{\hat{h^{*}}}^{+}{ }_{j, m}(\rho):=\left[Q_{j, m}^{+} h_{m}\right](\rho)=\frac{\Delta_{j, m}^{+}(\rho)}{\Delta^{+}(\rho)} \mathrm{e}^{\lambda_{j}^{+}(\rho)} \hat{h}_{m}^{+}(\rho) .
$$

Viewing $h_{j, m}^{*}$ as the inverse Fourier transform of ${\hat{h^{*}}}_{j, m}^{+}$, it is straightforward to see that for any $s \in \mathbb{R}$,

$$
\left\{\begin{array}{l}
h_{1} \in H_{0}^{(s+1) / 3}\left(\mathbb{R}^{+}\right) \Rightarrow h_{j, 1}^{*} \in H^{\frac{s+1}{3}}(\mathbb{R}), j=1,2,3, \\
h_{2} \in H_{0}^{s / 3}\left(\mathbb{R}^{+}\right) \Rightarrow h_{j, 2}^{*} \in H^{\frac{s+1}{3}}(\mathbb{R}), j=1,2,3, \\
h_{3} \in H_{0}^{(s-1) / 3}\left(\mathbb{R}^{+}\right) \Rightarrow h_{j, 3}^{*} \in H^{\frac{s+1}{3}}(\mathbb{R}), j=1,2,3 .
\end{array}\right.
$$

The proof is completed by the same arguments presented in [5] for their proofs of Propositions 2.7-2.9.

Now we consider the following IBVP of the linear KdV equation with variable coefficients.

$$
\left\{\begin{array}{l}
v_{t}+v_{x}+v_{x x x}+(a v)_{x}=f, \quad x \in(0, L), t>0, \\
v(x, 0)=\phi(x), \\
v(0, t)=h_{1}(t), v_{x}(L, t)=h_{2}(t), v_{x x}(L, t)=h_{3}(t), \quad t \geq 0
\end{array}\right.
$$

were $a=a(x, t)$ is a given function.

Proposition 2.4. Let $T>0$ be given and assume that $a \in Z_{0, T}$. Then for any $(\phi, \boldsymbol{h}) \in D_{0, T}$ and $f \in$ $L^{1}\left(0, T ; L^{2}(0, L)\right)$, the IBVP $(2.13)$ admits a unique solution $v \in Z_{0, T}$. Moreover, there exists a constant $C>0$ depending only on $T$ and $\|a\|_{Z_{0, T}}$ such that

$$
\|v\|_{Z_{0, T}} \leq C\left(\|(\phi, \boldsymbol{h})\|_{D_{0, T}}+\|f\|_{L^{1}\left(0, T ; L^{2}(0, L)\right)}\right) .
$$


Proof. Let $\eta>0$ and $0<\theta \leq \max \{1, T\}$ be constants to be determined. Set

$$
S_{\theta, \eta}=\left\{w \in Z_{0, \theta}, \quad\|w\|_{Z_{0, \theta}} \leq \eta\right\} .
$$

For given $(\phi, \boldsymbol{h}) \in \mathcal{D}_{0, T}$ define a map on $S_{\theta, \eta}$ by

$$
v=\Gamma(w)
$$

with $v$ being the unique solution of the IBVP

$$
\left\{\begin{array}{l}
v_{t}+v_{x}+v_{x x x}=-(a w)_{x}+f, \quad x \in(0, L), t \in(0, T) \\
v(x, 0)=\phi(x), \\
v(0, t)=h_{1}(t), \quad v_{x}(L, t)=h_{2}(t), \quad v_{x x}(L, t)=h_{3}(t)
\end{array}\right.
$$

for $w \in S_{\theta, \eta}$. Using Propositions 2.2 and 2.3, we have

$$
\begin{aligned}
\|\Gamma(w)\|_{Z_{0, \theta}} & \leq c_{1}\|(\phi, \boldsymbol{h})\|_{\mathcal{D}_{0, T}}+c_{1}\|f\|_{L^{1}\left(0, T ; L^{2}(0, L)\right)}+c_{1}\left\|(a w)_{x}\right\|_{L^{1}\left(0, \theta ; L^{2}(0, L)\right)} \\
& \leq c_{1}\|(\phi, \boldsymbol{h})\|_{\mathcal{D}_{0, T}}+c_{1}\|f\|_{L^{1}\left(0, T ; L^{2}(0, L)\right)}+c_{1}\left(\theta^{\frac{1}{3}}+\theta^{\frac{1}{2}}\right)\|a\|_{Z_{0, T}}\|w\|_{Z_{0, \theta}} .
\end{aligned}
$$

Here we have used the bilinear estimate $(c f .[5,34])$

$$
\int_{0}^{T}\left\|(q p)_{x}\right\|_{L^{2}(0, L)} \mathrm{d} t \leq C\left(T^{\frac{1}{2}}+T^{\frac{1}{3}}\right)\|q\|_{Z_{0, T}}\|q\|_{Z_{0, T}}
$$

for any $p, q \in Z_{0, T}$.

If we choose $\eta$ and $\theta$ such that

$$
\eta=2 c_{1}\left(\|(\phi, \boldsymbol{h})\|_{\mathcal{D}_{0, T}}+\|f\|_{L^{1}\left(0, T ; L^{2}(0, L)\right)}\right)
$$

and

$$
c_{1}\left(\theta^{\frac{1}{3}}+\theta^{\frac{1}{2}}\right)\|a\|_{Z_{0, T}} \leq \frac{1}{2} .
$$

Then we have

$$
\|\Gamma(w)\|_{Z_{0, \theta}} \leq \eta
$$

for any $w \in S_{\theta, \eta}$. Moreover, for any $w_{1}, w_{2} \in S_{\theta, \eta}$, one has

$$
\left\|\Gamma\left(w_{1}\right)-\Gamma\left(w_{2}\right)\right\|_{Z_{0, \theta}} \leq \frac{1}{2}\left\|w_{1}-w_{2}\right\|_{Z_{0, \theta}} .
$$

Thus, $\Gamma$ is a contraction on $S_{\theta, \eta}$ whose fixed point $u \in Z_{0, \theta}$ is the desired solution of the IBVP (2.13) which exists in the time interval $(0, \theta)$. As $\theta$ only depends on $\|a\|_{Z_{0, T}}$, by a standard argument the time interval $(0, \theta)$ in which the solution exists can be extended to $(0, T)$ so that $u \in Z_{0, T}$.

Proposition 2.4 will be sufficient for us to obtain the local well-posedness of the IBVP (1.1) in the space $H^{s}(0, L)$ for $s \geq 0$. However, in order to use Bourgain space analysis to obtain well-posedness in the space $H^{s}(0, L)$ with $s<0$, we need to extend the problem posed on the finite domain $(0, L) \times(0, T)$ to an equivalent problem posed on the whole plane $\mathbb{R} \times \mathbb{R}$.

Recall the solution of the following linear $\mathrm{KdV}$ equation,

$$
\left\{\begin{array}{l}
v_{t}+v_{x}+v_{x x x}=0, \quad x \in \mathbb{R}, t \in \mathbb{R}^{+}, \\
v(x, 0)=\psi(x)
\end{array}\right.
$$


has the explicit form

$$
v(x, t)=\left[W_{\mathbb{R}}(t)\right] \psi(x)=c \int_{\mathbb{R}} \mathrm{e}^{\mathrm{i}\left(\xi^{3}-\xi\right) t} \mathrm{e}^{\mathrm{i} x \xi} \hat{\psi}(\xi) \mathrm{d} \xi
$$

where $\hat{\psi}$ denotes the Fourier transform of $\psi$. Taking advantage of this simplicity as it is done in [5], rewrite $W_{0}(t)$ in terms of $W_{\mathbb{R}}(t)$ and $W_{b d r}(t)$. For any $\phi \in H^{s}(0, L)$, let $\phi^{*} \in H^{s}(\mathbb{R})$ be its standard extension from $(0, L)$ to $\mathbb{R}$. Let $v=v(x, t)$ be the solution of

$$
\left\{\begin{array}{l}
v_{t}+v_{x}+v_{x x x}=0, \quad x \in \mathbb{R}, t \geq 0 \\
v(x, 0)=\phi^{*}
\end{array}\right.
$$

and set $g_{1}(t)=v(0, t), g_{2}(t)=v_{x}(L, t)$ and $g_{3}(t)=v_{x x}(L, t), \boldsymbol{g}=\left(g_{1}, g_{2}, g_{3}\right)$ and

$$
v_{\boldsymbol{g}}=v_{\boldsymbol{g}}(x, t)=\left[W_{b d r}(t) \boldsymbol{g}\right](x),
$$

which is the corresponding solution of the nonhomogeneous boundary-value problem (2.11) with boundary data $h_{j}(t)=g_{j}(t)$ for $j=1,2,3$ and $t \geq 0$. Then $v(x, t)-v_{\boldsymbol{g}}$ solves the IBVP $(2.9)$ with $f \equiv 0$. This then leads us to a particular representation of $W_{0}(t)$ in terms of $W_{b d r}(t)$ and $W_{\mathbb{R}}(t)$.

Let $E: H^{s}(0, L) \rightarrow H^{s}(\mathbb{R})$ be a standard extension operator from $H^{s}(0, L)$ to $H^{s}(\mathbb{R})$.

Lemma 2.5. Given $s \in \mathbb{R}$ and $\phi \in H^{s}(0, L)$, let $\phi^{*}=E \phi$. Then

$$
W_{0}(t) \phi=W_{\mathbb{R}}(t) \phi^{*}-W_{b d r}(t) \boldsymbol{g}
$$

for any $t>0$ and $x \in(0, L)$, where $\boldsymbol{g}=\left(g_{1}, g_{2}, g_{3}\right)$ is obtained from the traces of

$$
p(x, t)=W_{\mathbb{R}}(t) \phi^{*}
$$

at $x=0, L$, i.e.,

$$
p(0, t)=g_{1}(t), \quad p_{x}(L, t)=g_{2}(t), \quad p_{x x}(L, t)=g_{3}(t) .
$$

The solution of the non-homogeneous initial boundary-value problem

$$
\left\{\begin{array}{l}
v_{t}+v_{x}+v_{x x x}=f, \quad x \in(0, L), t \geq 0, \\
v(x, 0)=0, \\
v(0, t)=0, \quad v_{x}(L, t)=0, \quad v_{x x}(L, t)=0
\end{array}\right.
$$

can also be expressed in terms of $W_{\mathbb{R}}(t)$ and $W_{b d r}(t)$.

Lemma 2.6. If $f^{*}(., t)=E f(., t)$, with $E$ as was defined before the extension of $f$ from $[0, L] \times \mathbb{R}^{+} \rightarrow \mathbb{R} \times \mathbb{R}^{+}$, then the solution $u$ of the extended problem (2.17) is

$$
v(x, t)=\int_{0}^{t} W_{0}(t-\tau) f(\cdot, \tau) \mathrm{d} \tau=\int_{0}^{t} W_{\mathbb{R}}(t-\tau) f^{*}(., \tau) \mathrm{d} \tau-\left[W_{b d r}(t) \boldsymbol{v}\right](x)
$$

for any $x \in(0, L)$ and $t \geq 0$ where $\boldsymbol{v} \equiv \boldsymbol{v}(t)=\left(v_{1}(t), v_{2}(t), v_{3}(t)\right)$ is the appropriate boundary traces of

$$
q(x, t)=\int_{0}^{t} W_{\mathbb{R}}(t-\tau) f^{*}(\cdot, \tau) \mathrm{d} \tau
$$

at $x=0, L$, i.e.

$$
v_{1}(t)=q(0, t), v_{2}(t)=q_{x}(L, t), v_{3}(t)=q_{x x}(L, t) .
$$


Lemmas 2.5 and 2.6 are validated when $x \in(0, L)$ and $t \geq 0$ since some of the operators that we have constructed are defined only in this interval. The only operator that is defined in the whole line is $W_{\mathbb{R}}(t)$ for any values of $x$ and $t$. Also it should be noted that for equation (2.16) the first term on the right-hand side is defined for all $x \in \mathbb{R}$ but the second term on the right-hand side is defined just on $(0, L)$. In order to employ the use of Bourgain spaces, we need to extend the second operator of the right hand side.

Recall that

$$
W_{b d r}(t) \boldsymbol{h}=\sum_{j, m=1}^{3} W_{j, m} h_{j}
$$

and each $W_{j, m} h_{j}$ is either of the form.

$$
\left[U_{b d r}^{1}(t)\right] h(x)=\frac{1}{2 \pi} \mathfrak{R e} \int_{1}^{\infty} \mathrm{e}^{\mathrm{i} t\left(\mu^{3}-\mu\right)} \mathrm{e}^{\frac{-\sqrt{3 \mu^{2}-4}-i \mu}{2} x}\left(3 \mu^{2}-1\right) \hat{h}(\mu) \mathrm{d} \mu
$$

or of the form

$$
\left[U_{b d r}^{2}(t)\right] h(x)=\frac{1}{2 \pi} \mathfrak{R e} \int_{1}^{\infty} \mathrm{e}^{\mathrm{i} t\left(\mu^{3}-\mu\right)} \mathrm{e}^{\mathrm{i} \mu x}\left(3 \mu^{2}-1\right) \hat{h}(\mu) \mathrm{d} \mu
$$

where $\hat{h}(\mu)=h\left(i\left(\mu^{3}-\mu\right)\right.$ ) (from Lem. 2.1). Therefore by the extension method introduced in [7], the operator $W_{b d r}(t)$ can be extended as $\mathcal{W}_{b d r}(t)$ with

$$
\left[\mathcal{W}_{b d r}(t) \boldsymbol{h}\right](x, t)
$$

defined for any $t, x \in \mathbb{R}$ and

$$
\left[\mathcal{W}_{b d r}(t) \boldsymbol{h}\right](x, t)=\left[W_{b d r} \boldsymbol{h}\right](x, t) \text { for any }(x, t) \in(0, L) \times(0, T) .
$$

Moreover, the following estimates hold.

Proposition 2.7. For given $\alpha>\frac{1}{2}$ and $(b, s)$ such that $s \leq 0$ and $b<1 / 2$ satisfying

$$
0 \leq b<1 / 2-s / 3
$$

there exists a constant $C$ such that for any $T>0$ and any $\boldsymbol{h} \in \mathbb{H}^{s}(0, T)$,

$$
\mathcal{W}_{b d r} \boldsymbol{h} \in \mathcal{X}_{s, b}^{\alpha, T}
$$

and

$$
\left\|\mathcal{W}_{b d r} \boldsymbol{h}\right\|_{\mathcal{X}_{s, b}^{\alpha, T}} \leq C\|\boldsymbol{h}\|_{\mathbb{H}^{s}(0, T)}
$$

Several key lemmas for this research from the literature regarding varying estimates will now be presented. The first lemma gives the Bourgain estimates of the operator $W_{\mathbb{R}}(t)$ established in $[7,20,26,31]$.

Lemma 2.8. Let $-\infty<s<\infty, 0<b \leq 1, \frac{1}{2}<\alpha \leq 1$, and $\psi \in C_{0}^{\infty}(\mathbb{R})$ be given. There exists a constant $C$ depending only on $s, \alpha, b$ and $\psi$ such that

$$
\left\|\psi(t) W_{\mathbb{R}}(t) \phi\right\|_{\mathcal{X}_{s, b}^{\alpha}} \leq C\|\phi\|_{H^{s}(\mathbb{R})}
$$

and

$$
\left\|\psi(t) \int_{0}^{t} W_{\mathbb{R}}\left(t-t^{\prime}\right) f\left(t^{\prime}\right) \mathrm{d} t^{\prime}\right\|_{\mathcal{X}_{s, b}^{\alpha}} \leq C_{\delta}\|f\|_{Y_{s, 1-b}^{1-\alpha}} .
$$

The next two lemmas present the spatial trace estimates for $W_{\mathbb{R}}(t) \phi$ and $\int_{0}^{t} W_{\mathbb{R}}\left(t-t^{\prime}\right) f\left(\cdot, t^{\prime}\right) \mathrm{d} t^{\prime}$ are presented. Their proofs can be found in $[20,26]$. 
Lemma 2.9. Let $s \in[-1,2]$ be given. There exists a constant $C$ depending only on s such that

$$
\begin{aligned}
& \sup _{x \in \mathbb{R}}\left\|W_{\mathbb{R}}(t) \phi\right\|_{H_{t}^{\frac{s+1}{3}}(\mathbb{R})} \leq C\|\phi\|_{H^{s}(\mathbb{R})}, \\
& \sup _{x \in \mathbb{R}}\left\|\partial_{x} W_{\mathbb{R}}(t) \phi\right\|_{H_{t}^{\frac{s}{3}(\mathbb{R})}} \leq C\|\phi\|_{H^{s}(\mathbb{R})}
\end{aligned}
$$

and

$$
\sup _{x \in \mathbb{R}}\left\|\partial_{x x} W_{\mathbb{R}}(t) \phi\right\|_{H_{t}^{\frac{s-1}{3}}(\mathbb{R})} \leq C\|\phi\|_{H^{s}(\mathbb{R})} .
$$

Lemma 2.10. Let $0 \leq b<1 / 2,-1 \leq s \leq 2, f \in Y_{s, b}$ and

$$
w(x, t)=\int_{0}^{t} W_{\mathbb{R}}\left(t-t^{\prime}\right) f\left(\cdot, t^{\prime}\right) \mathrm{d} t^{\prime} .
$$

There exists a constant $C$ depending only on $b, s$ and $\psi$ such that

$$
\begin{aligned}
& \sup _{x \in \mathbb{R}}\|\psi(\cdot) w(x, \cdot)\|_{H_{t}^{\frac{s+1}{3}}(\mathbb{R})} \leq C\|f\|_{Y_{s, b}}, \\
& \sup _{x \in \mathbb{R}}\left\|\psi(\cdot) w_{x}(x, \cdot)\right\|_{H_{t}^{\frac{s}{3}}(\mathbb{R})} \leq C\|f\|_{Y_{s, b}}
\end{aligned}
$$

and

$$
\sup _{x \in \mathbb{R}}\left\|\psi(\cdot) w_{x x}(x, \cdot)\right\|_{H_{t}^{\frac{s-1}{3}}(\mathbb{R})} \leq C\|f\|_{Y_{s, b}} .
$$

The last lemma gives the crucial bilinear estimate established in [20,26,31].

Lemma 2.11. Given $s>-\frac{3}{4}$, there exist $b=b(s)<\frac{1}{2}, \alpha=\alpha(s)>\frac{1}{2}$ and $C, \mu>0$ such that

$$
\left\|\partial_{x}(u v)\right\|_{Y_{s, b}^{\alpha}} \leq C T^{\mu}\|u\|_{X_{s, b}^{\alpha}}\|v\|_{X_{s, b}^{\alpha}}
$$

for any $u, v \in X_{s, b}^{\alpha}$ with compact support in $[-T, T]$.

\section{NonlineAr PROBLEM}

In this section, we consider the well-posedness of the following nonlinear problem in the space $H^{s}(0, L)$.

$$
\left\{\begin{array}{l}
v_{t}+v_{x}+v v_{x}+v_{x x x}=0, \quad x \in(0, L), t \geq 0 \\
v(x, 0)=\phi(x), \\
v(0, t)=h_{1}(t), v_{x}(L, t)=h_{2}(t), v_{x x}(L, t)=h_{3}(t) .
\end{array}\right.
$$

First we consider its well-posedness in the space $H^{s}(0, L)$ for $s \geq 0$. Recall that for given $s \geq 0$ and $T>0$,

$$
D_{s, T}:=H^{s}(0, L) \times H^{\frac{s+1}{3}}(0, T) \times H^{\frac{s}{3}}(0, T) \times H^{\frac{s-1}{3}}(0, T)
$$

and

$$
Z_{s, T}:=C\left([0, T] ; H^{s}(0, L)\right) \cap L^{2}\left(0, T ; H^{s+1}(0, L)\right)
$$

In addition, let

$$
\mathcal{Z}_{s, T}:=Z_{s, T} \cap H^{\frac{s}{3}}\left(0, T ; H^{1}(0, L)\right) .
$$

Note that

$$
\mathcal{Z}_{0, T}=Z_{0, T}
$$

The following theorems will now be proven. 
Theorem 3.1. Let $T>0, r>0$ and $0 \leq s \leq 3$ be given with $s \neq \frac{2 j+1}{2}$ for $j=0,1,2$. There exists $a T^{*} \in(0, T]$ depending only on $r$ and $T$ such that for any s-compatible $(\phi, \boldsymbol{h}) \in D_{s, T}$ satisfying

$$
\|(\phi, h)\|_{D_{0, T}} \leq r
$$

the IBVP (3.1) admits a unique solution

$$
v \in \mathcal{Z}_{s, T^{*}}
$$

Moreover, the corresponding solution map is Lipschitz continuous.

Proof. The proof is divided into three steps.

Step 1. Consider the case of $s=0$.

Let $\eta>0$ and $0<T^{*} \leq \min \{1, T\}$ be constants to be determined. Set

$$
S_{T^{*}, \eta}=\left\{w \in Z_{0, T^{*}}, \quad\|w\|_{Z_{0, T^{*}}} \leq \eta\right\} .
$$

For given $(\phi, \boldsymbol{h}) \in D_{0, T}$ with

$$
\|(\phi, h)\|_{D_{0, T}} \leq r
$$

define a nonlinear map on $S_{T^{*}, \eta}$ by

$$
v=\Gamma(w)
$$

with $v$ being the unique solution of the IBVP

$$
\left\{\begin{array}{l}
v_{t}+v_{x}+v_{x x x}=-w w_{x}, \quad x \in(0, L), t>0 \\
v(x, 0)=\phi(x), \\
v(0, t)=h_{1}(t), v_{x}(L, t)=h_{2}(t), v_{x x}(L, t)=h_{3}(t), \quad t \geq 0
\end{array}\right.
$$

for $w \in S_{T^{*}, \eta}$. As in the proof of Proposition 2.4, using Propositions 2.2 and 2.3 yields that

$$
\begin{aligned}
\|\Gamma(w)\|_{Z_{0, T^{*}}} & \leq c_{1}\|(\phi, \boldsymbol{h})\|_{D_{0, T}}+c_{1}\left\|w w_{x}\right\|_{L^{1}\left(0, T^{*} ; L^{2}(0, L)\right)} \\
& \leq c_{1}\|(\phi, h)\|_{D_{0, T}}+c_{1} c_{2}\left(T^{*}\right)^{\frac{1}{3}}\|w\|_{Z_{0, T^{*}}}^{2} .
\end{aligned}
$$

where $c_{j}, j=1,2$ are constants independent of $w$ and $(\phi, \boldsymbol{h})$. If we choose $\eta$ and $T^{*}$ such that

$$
\eta=2 c_{1}\|(\phi, \boldsymbol{h})\|_{D_{0, T}} \leq 2 c_{1} r
$$

and

$$
4 c_{2} c_{1}^{2}\left(T^{*}\right)^{\frac{1}{3}} r \leq \frac{1}{2}
$$

Then, for any $w \in S_{T^{*}, \eta}$, we have

$$
\begin{aligned}
\|\Gamma(w)\|_{Z_{0, T^{*}}} & \leq \frac{1}{2} \eta+c_{2} c_{1}\left(T^{*}\right)^{\frac{1}{3}} \eta^{2} \\
& \leq \frac{1}{2} \eta+2 c_{2} c_{1}^{2}\left(T^{*}\right)^{\frac{1}{3}} r \eta \\
& \leq \frac{1}{2} \eta+\frac{1}{2} \eta=\eta .
\end{aligned}
$$


Moreover, for any $w_{1}, w_{2} \in S_{T^{*}, \eta}$, one has

$$
\begin{aligned}
\left\|\Gamma\left(w_{1}\right)-\Gamma\left(w_{2}\right)\right\|_{Z_{0, T^{*}}} & \leq \frac{1}{2} c_{1}\left\|\left(\left(w_{1}+w_{2}\right)\left(w_{1}-w_{2}\right)\right)_{x}\right\|_{L^{1}\left(0, T^{*} ; L^{2}(0, L)\right)} \\
& \leq c_{2} c_{1}\left\|w_{1}+w_{2}\right\|_{Z_{0, T^{*}}}\left\|w_{1}-w_{2}\right\|_{Z_{0, T^{*}}} \\
& \leq 4 c_{2} c_{1}^{2}\left(T^{*}\right)^{\frac{1}{3}} r\left\|w_{1}-w_{2}\right\|_{Z_{0, T^{*}}} \\
& \leq \frac{1}{2}\left\|w_{1}-w_{2}\right\|_{Z_{0, T^{*}}} .
\end{aligned}
$$

Thus, $\Gamma$ is a contraction on $S_{T^{*}, \eta}$ whose fixed point $u \in Z_{0, T^{*}}$ solves the IBVP (3.1) which satisfies

$$
\|u\|_{Z_{0, T *}} \leq 2 c_{1}\|(\phi, h)\|_{D_{0, T}} .
$$

Moreover, for $(\phi, \boldsymbol{h}),(\psi, \boldsymbol{g}) \in D_{0, T}$ with

$$
\|(\phi, \boldsymbol{h})\|_{D_{0, T}} \leq r, \quad\|(\psi, \boldsymbol{g})\|_{D_{0, T}} \leq r,
$$

let $u_{1}$ and $u_{2}$ be the corresponding solutions of the IBVP (3.1). Then $w=u_{1}-u_{2}$ solves the following linear system

$$
\left\{\begin{array}{l}
w_{t}+w_{x}+(a w)_{x}+w_{x x x}=0, \quad x \in(0, L), t \in\left(0, T^{*}\right) \\
w(x, 0)=\phi(x)-\psi(x), \quad x \in(0, L), \\
w(0, t)=h_{1}(t)-g_{1}(t) . \quad w_{x}(L, t)=h_{2}(t)-g_{2}(t), \quad w_{x x}(L, t)=h_{3}(t)-g_{3}(t)
\end{array}\right.
$$

where $a=\frac{1}{2}\left(u_{1}+u_{2}\right)$. Applying Proposition 2.4 yields that

$$
\left\|u_{1}-u_{2}\right\|_{Z_{0, T^{*}}} \leq C\|(\phi, \boldsymbol{h})-(\psi, \boldsymbol{g})\|_{D_{0, T}}
$$

where the constant $C$ depends only on $\left\|u_{1}+u_{2}\right\|_{Z_{0, T^{*}}}$ and therefore only depends on $\|(\phi, h)\|_{D_{0, T}}+\|(\psi, \boldsymbol{g})\|_{D_{0, T}}$.

Step 2. Consider the case of $s=3$.

For given 3-compatible $(\phi, \boldsymbol{h}) \in D_{3, T}$, let $u \in Z_{0, T^{*}}$ be the corresponding solution given in Step 1. Set $v=u_{t}$. Then $v$ solves the following IBVP of the linear KdV equation with variable coefficient $a(x, t)=u(x, t)$.

$$
\left\{\begin{array}{l}
v_{t}+v_{x}+v_{x x x}+(a v)_{x}=0, \quad x \in(0, L), t>0 \\
v(x, 0)=\phi^{*}(x) \\
v(0, t)=h_{1}^{\prime}(t), v_{x}(L, t)=h_{2}^{\prime}(t), v_{x x}(L, t)=h_{3}^{\prime}(t), \quad t \geq 0
\end{array}\right.
$$

where

$$
\phi^{*}(x)=-\phi^{\prime}(x)-\phi(x) \phi^{\prime}(x)-\phi^{\prime \prime \prime}(x) .
$$

Applying Proposition 2.4 yields that $v$ belongs to the space $Z_{0, T^{*}}$ and satisfies

$$
\|v\|_{Z_{0, T^{*}}} \leq C\left\|\left(\phi^{*}, \boldsymbol{h}^{\prime}\right)\right\|_{D_{0, T}}
$$

where the constant $C$ depends only on $\|a\|_{Z_{0, T^{*}}}$ and is therefore only depends on $\|(\phi, \boldsymbol{h})\|_{D_{0, T}}$. It then follows from the relation

$$
u_{x x x}=-u_{t}-u u_{x}-u_{x}
$$

that $u \in \mathcal{Z}_{3, T *}$ and

$$
\|u\|_{\mathcal{Z}_{3, T^{*}}} \leq C\|(\phi, \boldsymbol{h})\|_{D_{3, T}}
$$

where $C$ is a constant depending only on $\|(\phi, \boldsymbol{h})\|_{D_{0, T}}$. 
Step 3. Consider the case of $0<s<3$.

For given $r>0$, we define operator $I_{r}: D_{0, T} \rightarrow D_{0, T}$ by

$$
I_{r}(\phi, \boldsymbol{h})= \begin{cases}(\phi, \boldsymbol{h}) & \text { if }\|(\phi, \boldsymbol{h})\|_{D_{0, T}}<r \\ r(\phi, \boldsymbol{h}) /\|(\phi, \boldsymbol{h})\|_{D_{0, T}} & \text { if }\|(\phi, \boldsymbol{h})\|_{D_{0, T}} \geq r\end{cases}
$$

for any $(\phi, \boldsymbol{h}) \in D_{0, T}$. Then for any $(\phi, \boldsymbol{h}) \in D_{0, T}$,

$$
\left\|I_{r}(\phi, h)\right\|_{D_{0, T}} \leq r
$$

and for any $(\phi, \boldsymbol{h}) \in D_{3, T}$,

$$
\left\|I_{r}(\phi, \boldsymbol{h})\right\|_{D_{3, T}} \leq\|(\phi, \boldsymbol{h})\|_{D_{3, T}} .
$$

Furthermore, for any $(\phi, \boldsymbol{h}),(\psi, \boldsymbol{g}) \in D_{0, T}$,

$$
\left\|I_{r}(\phi, \boldsymbol{h})-I_{r}(\psi, \boldsymbol{g})\right\|_{D_{0, T}} \leq C\|(\phi, \boldsymbol{h})-(\psi, \boldsymbol{g})\|_{D_{0, T}}
$$

where $C$ is a constant depending only on $\|(\phi, \boldsymbol{h})\|_{D_{0, T}}$ and $\|(\psi, \boldsymbol{g})\|_{D_{0, T}}$.

For given $r>0$ and $T>0$ let $T^{*}$ be as given in Step 1. Set

$$
\begin{gathered}
B_{0}^{1}=D_{0, T}, \quad B_{1}^{1}=Z_{0, T^{*}}, \\
B_{0}^{2}=\left\{(\phi, \boldsymbol{h}) \in D_{3, T} ; \quad(\phi, \boldsymbol{h}) \text { is 3-compatible }\right\}
\end{gathered}
$$

and $B_{1}^{2}=\mathcal{Z}_{3, T *}$. Note that

$$
B_{0}^{2} \subset B_{0}^{1}
$$

and $B_{0}^{2}$ is a closed subspace of $D_{3, T}$ and is therefore a Banach space.

Define the operator $K_{r}: B_{0}^{j} \rightarrow B_{1}^{j}, j=1,2$ by

$$
K_{r}(\phi, \boldsymbol{h}):=u
$$

for any $(\phi, \boldsymbol{h}) \in B_{0}^{j}$, where $u \in B_{1}^{j}$ is the solution of the $\operatorname{IBVP}(3.1)$ with initial-boundary datum $I_{r}(\phi, \boldsymbol{h})$.

From Step 1, for any $\left(\phi_{1}, \boldsymbol{h}_{1}\right),\left(\phi_{2}, \boldsymbol{h}_{2}\right) \in B_{0}^{1}$,

$$
\left\|K_{r}\left(\phi_{1}, \boldsymbol{h}_{1}\right)-K_{r}\left(\phi_{2}, \boldsymbol{h}_{2}\right)\right\|_{B_{1}^{1}} \leq C_{1}\left\|I_{r}\left(\phi_{1}, \boldsymbol{h}_{1}\right)-I_{r}\left(\phi_{2}, \boldsymbol{h}_{2}\right)\right\|_{B_{0}^{1}} \leq C_{2}\left\|\left(\phi_{1}, \boldsymbol{h}_{1}\right)-\left(\phi_{2}, \boldsymbol{h}_{2}\right)\right\|_{B_{0}^{1}}
$$

where $C_{2}$ depends only on $\left\|\left(\phi_{1}, \boldsymbol{h}_{1}\right)\right\|_{B_{0}^{1}}+\left\|\left(\phi_{2}, \boldsymbol{h}_{2}\right)\right\|_{B_{0}^{1}}$.

From Step 2, we see that for any $(\phi, \boldsymbol{h}) \in B_{0}^{2}$,

$$
\left\|K_{r}(\phi, \boldsymbol{h})\right\|_{B_{1}^{2}} \leq C_{3}\left\|I_{r}(\phi, \boldsymbol{h})\right\|_{B_{0}^{2}} \leq C_{4}\|(\phi, \boldsymbol{h})\|_{B_{0}^{2}}
$$

where $C_{4}$ depends only on $\|(\phi, h)\|_{B_{0}^{1}}$. Thus, invoking Tartar's nonlinear interpolation theory yields that for $0<s<3$ with $s \neq \frac{2 j+1}{2}, j=0,1,2$, and $s$-compatible $(\phi, \boldsymbol{h}) \in D_{s, T}, K_{r}(\phi, \boldsymbol{h}) \in \mathcal{Z}_{s, T^{*}}$ and

$$
\left\|K_{r}(\phi, \boldsymbol{h})\right\|_{\mathcal{Z}_{s, T^{*}}} \leq C\|(\phi, \boldsymbol{h})\|_{D_{s, T}}
$$

where $C$ only depends on $\|(\phi, \boldsymbol{h})\|_{D_{0, T}}$. Note that $I_{r}(\phi, \boldsymbol{h})=(\phi, \boldsymbol{h})$ when

$$
\|(\phi, h)\|_{D_{0, T}} \leq r .
$$

Therefore $u=K_{r}(\phi, \boldsymbol{h}) \in \mathcal{Z}_{s, T^{*}}$ solves the $\operatorname{IBVP}(3.1)$ when $s$-compatible $(\phi, \boldsymbol{h}) \in D_{s, T}$ satisfying $\|(\phi, h)\|_{D_{0, T}} \leq r$. 
To prove the well-posed of the IBVP (3.1) in the space $H^{s}(0, L)$ for $s>3$, we first reconsider the wellposedness of the IBVP of the linear KdV equation with variable coefficients $a=a(x, t)$ :

$$
\left\{\begin{array}{l}
u_{t}+u_{x}+(a u)_{x}+u_{x x x}=f, \quad x \in(0, L), t \in(0, T), \\
u(x, 0)=\phi(x), \quad x \in(0, L), \\
u(0, t)=h_{1}(t), \quad u_{x}(L, t)=h_{2}(t), \quad u_{x x}(L, t)=h_{3}(t) .
\end{array}\right.
$$

Proposition 3.2. Let $s \in[0,3]$ and $T>0$ be given. Assume that $a \in \mathcal{Z}_{3, T}$. Then for any s-compatible $(\phi, \boldsymbol{h}) \in$ $D_{s, T}$ and $f \in W^{1,1}\left(0, T ; L^{2}(0, L)\right) \cap L^{2}\left(0, T ; H^{\frac{s}{3}}(0, L)\right)$, the IBVP (3.7) admits a unique solution $u \in \mathcal{Z}_{3, T}$. Moreover, there exists a constant $C>0$ depending only on $T$ and $\|a\|_{\mathcal{Z}_{3, T}}$ such that

$$
\|u\|_{\mathcal{Z}_{s, T}} \leq C\left(\|(\phi, \boldsymbol{h})\|_{D_{s, T}}+\|f\|_{W^{\frac{s}{3}}\left(0, T ; L^{2}(0, L)\right)}+\|f\|_{L^{2}\left(0, T ; H^{\frac{s}{3}}(0, L)\right)}\right) .
$$

Proof. When $s=0$, it follows from Proposition 2.4 that for any $(\phi, \boldsymbol{h}) \in D_{0, T}$, the IBVP (3.7) admits a unique solution $u \in Z_{0, T}$ satisfying

$$
\|u\|_{Z_{0, T}} \leq C\|(\phi, \boldsymbol{h})\|_{D_{0, T}} .
$$

When $s=3$, let $v=u_{t}$. Then $v$ solves

$$
\left\{\begin{array}{l}
v_{t}+v_{x}+(a v)_{x}+v_{x x x}=-\left(a_{t} u\right)_{x}+f_{t}, \quad x \in(0, L), \quad t \in(0, T), \\
u(x, 0)=f(x, 0)-\phi^{\prime \prime \prime}(x)-\phi^{\prime}(x)-(a(x, 0) \phi(x))^{\prime}, \quad x \in(0, L), \\
v(0, t)=h_{1}^{\prime}(t), \quad v_{x}(L, t)=h_{2}^{\prime}(t), \quad v_{x x}(L, t)=h_{3}^{\prime}(t)
\end{array}\right.
$$

Note that both $f_{t}$ and $\left(a_{t} u\right)_{x}$ belong to the space $L^{1}\left(0, T ; L^{2}(0, L)\right)$. Applying Proposition 2.4 again yields that $u_{t}=v \in Z_{0, T}$. Because of

$$
u_{x x x}=u_{t}-u_{x}-(a u)_{x}+f
$$

and $f \in L^{2}\left(0, H^{1}(0, L)\right)$, we arrive at $u \in \mathcal{Z}_{3, T}$ and

$$
\|u\|_{\mathcal{Z}_{3, T}} \leq C\left(\|(\phi, \boldsymbol{h})\|_{D_{3, T}}+\|f\|_{W^{1,1}\left(0, T ; L^{2}(0, L)\right)}+\|f\|_{L^{2}\left(0, T ; H^{1}(0, L)\right)}\right)
$$

where $C$ depends only on $\|a\|_{\mathcal{Z}_{3, T}}$. The proof is then completed by invoking standard linear interpolation theory.

We now show that the IBVP (3.1) is well-posed in the space $H^{s}(0, L)$ for $s \geq 3$.

Theorem 3.3. Let $T>0, r>0$ and $s \geq 3$ be given with $s \neq \frac{2 j+1}{2}$ for $j=3,4, \ldots$ There exists a $T^{*} \in(0, T]$ depending only on $r$ and $T$ such that for any s-compatible $(\phi, \boldsymbol{h}) \in D_{s, T}$ with

$$
\|(\phi, h)\|_{D_{0, T}} \leq r
$$

the IBVP (3.1) admits a unique solution $u \in \mathcal{Z}_{s, T^{*}}$ with

$$
\partial_{t}^{j} u \in \mathcal{Z}_{s-3 j, T^{*}}
$$

for $j=1,2, \cdots,\left[\frac{s}{3}\right]$. Moreover, the corresponding solution map is Lipschitz continuous.

${ }^{9}$ When $0 \leq s \leq 3,(\phi, \boldsymbol{h})$ is $s$-compatible with respect to the IBVP (3.7) if and only if $(\phi, \boldsymbol{h})$ is $s$-compatible with respect to the $\operatorname{IBVP}(3.1)$. 
Proof. For $s \geq 3$ and $s$-compatible $(\phi, h)$ with

$$
\|(\phi, h)\|_{D_{0, T}} \leq r
$$

by Theorem 3.1, there exists a unique solution $u \in \mathcal{Z}_{3, T^{*}}$ for some $T^{*}>0$ depending only on $T$ and $r$. Set

$$
u^{(k)}=\partial_{t}^{k} u
$$

for $k=1,2, \cdots\left[\frac{s}{3}\right]$. Then $u^{(1)}$ solves

$$
\left\{\begin{array}{l}
u_{t}^{(1)}+u_{x}^{(1)}+\left(a u^{(1)}\right)_{x}+u_{x x x}^{(1)}=0, \quad x \in(0, L), \\
u^{(1)}(x, 0)=\phi_{1}(x), \quad x \in(0, L), \\
u^{(1)}(0, t)=h_{1}^{(1)}, \quad u_{x}^{(1)}(L, 0)=h_{2}^{(1)}(t), \quad u_{x x}^{(1)}(L, t)=h_{3}^{(1)}(t)
\end{array}\right.
$$

and $u^{(k)}$ solves

$$
\left\{\begin{array}{l}
u_{t}^{(k)}+u_{x}^{(k)}+\left(a u^{(k)}\right)_{x}+u_{x x x}^{(k)}=-\frac{1}{2} \sum_{j=1}^{k-1} \frac{k !}{j !(k-j) !}\left(u^{(j)} u^{(k-j}\right)_{x}, \quad x \in(0, L), \\
u^{(k)}(x, 0)=\phi_{k}(x), \quad x \in(0, L), \\
u^{(k)}(0, t)=h_{1}^{(k)}, \quad u_{x}^{(k)}(L, 0)=h_{1}^{(k)}(t), \quad u_{x x}^{(k)}(L, t)=h^{(k)}(t)
\end{array}\right.
$$

for $k=2, \cdots,\left[\frac{s}{3}\right]$, where $a(x, t)=u(x, t)$ and $\phi_{k}$ and $h^{(k)}$ is as defined in (1.5) and (1.4).

As $u \in \mathcal{Z}_{3, T^{*}}, u u_{x} \in L^{2}\left(0, T^{*} ; H^{1}(0, L)\right)$. Applying Proposition 3.2 to (3.8) yields that

$$
u^{(1)}=u_{t} \in \mathcal{Z}_{3, T^{*}}
$$

which imply further that

$$
u \in \mathcal{Z}_{6, T^{*}}
$$

Assume it holds for $k=N$ that

$$
u \in \mathcal{Z}_{3 N, T^{*}}, \quad u^{(N)} \in \mathcal{Z}_{3, T^{*}}
$$

Then

$$
-\frac{1}{2} \sum_{j=1}^{N} \frac{(N+1) !}{j !(N+1-j) !}\left(u^{(j)} u^{(N+1-j}\right)_{x}
$$

belongs to the space $L^{2}\left(0, T^{*} ; H^{1}(0, L)\right)$. Thus applying Proposition 3.2 yields that

$$
u^{(N+1)} \in \mathcal{Z}_{3, T^{*}}
$$

which implies further that

$$
u \in \mathcal{Z}_{3(N+1), T^{*}}
$$

The proof is then completed by induction.

Next we consider the well-posedness of the IVP (3.1) in the space $H^{s}(0, L)$ with $s<0$. We first rewrite the IBVP (3.1) in its integral form;

$$
v(t)=W_{0}(t) \phi+W_{b d r}(t) \boldsymbol{h}-\int_{0}^{t} W_{0}(t-\tau)\left(v v_{x}\right)(\tau) \mathrm{d} \tau .
$$


Theorem 3.4. Let $T>0, r>0$ and $-\frac{3}{4}<s<0$ be given. There exists a $T^{*} \in(0, T]$ and $\frac{1}{2}<\alpha \leq 1$ such that for any $(\phi, \boldsymbol{h}) \in D_{s, T}$ with

(3.10) admits a unique solution

$$
\|(\phi, h)\|_{D_{s, T}} \leq r
$$

$$
v \in \mathcal{X}_{s, \frac{1}{2}}^{\alpha, T^{*}}
$$

Moreover, the corresponding solution map is Lipschitz continuous.

The following lemmas are needed to prove Theorem 3.4.

Lemma 3.5. Let $T>0, s<0, \frac{1}{2}<\alpha \leq 1$ and $b \in(0,1)$ be given satisfying

$$
0<b<\frac{1}{2}-\frac{s}{3}
$$

For any $\phi \in H^{s}(0, L), W_{0}(t) \phi \in \mathcal{X}_{s, b}^{\alpha, T}$ and

$$
\left\|W_{0}(t) \phi\right\|_{\mathcal{X}_{s, b}^{\alpha, T}} \leq C\|\phi\|_{H^{s}(0, L)}
$$

where $C>0$ is independent of $\phi$.

Proof. It follows from Lemmas 2.5, and 2.8, 2.9, and Proposition 2.7.

Lemma 3.6. Assume that $-1 \leq s<1, \frac{1}{2}<\alpha \leq 1$ and $0<b<\frac{1}{2}$. For any $T>0$, there is a constant $C$ such that for any $f \in Y_{s, b}^{1-\alpha, T}$,

$$
u=\int_{0}^{t} W_{0}(t-\tau) f(\tau) \mathrm{d} \tau \in \mathcal{X}_{s, b}^{\alpha, T}
$$

and satisfies the inequality

$$
\|u\|_{\mathcal{X}_{s, b}^{\alpha, T}} \leq C\|f\|_{Y_{s, b}^{1-\alpha, T}}
$$

In addition, there exists a $b^{*} \in\left(0, \frac{1}{2}\right)$ such that if $f \in Y_{s, b^{*}}^{1-\alpha, T}$, then $u$ belongs to the space $\mathcal{X}_{s, \frac{1}{2}}^{\alpha, T}$ and satisfies the bound

$$
\|u\|_{\mathcal{X}_{s, \frac{1}{2}}^{\alpha, T}} \leq C\|f\|_{Y_{s, b^{*}}^{1-\alpha, T}}
$$

Proof. It follows from Lemmas 2.6, and 2.8, 2.10, and Proposition 2.7.

Lemma 3.7. Given $T>0, s>-\frac{3}{4}$, there exist $b=b(s)<\frac{1}{2}, \alpha=\alpha(s)>\frac{1}{2}$ and $C, \mu>0$ such that

$$
\left\|\partial_{x}(u v)\right\|_{Y_{s, b}^{\alpha, T}} \leq C T^{\mu}\|u\|_{\mathcal{X}_{s, b}^{\alpha, T}}\|v\|_{\mathcal{X}_{s, b}^{\alpha, T}}
$$

for any $u, v \in \mathcal{X}_{s, b}^{\alpha, T}$.

Proof. It follows from Lemma 2.11 directly.

Now we are at the position to present of the Proof of Theorem 3.4.

Proof of Theorem 3.4. For given $(\phi, \boldsymbol{h}) \in D_{s, T}$ and $s \in\left(-\frac{3}{4}, 0\right)$, let $\theta \in(0,1]$ to be determined. Let $\frac{1}{2}<\alpha \leq 1$ and $0<b<\frac{1}{2}$ be as chosen according to Lemma 3.7. Define $\Gamma: \mathcal{X}_{s, \frac{1}{2}}^{\alpha, \theta} \rightarrow \mathcal{X}_{s, \frac{1}{2}}^{\alpha, \theta}$ by

$$
\Gamma(\omega):=W_{0}(t) \phi+W_{b d r}(t) \boldsymbol{h}-\int_{0}^{t} W_{0}(t-\tau)\left(\omega \omega_{x}\right)(\tau) \mathrm{d} \tau
$$


By Lemmas 3.5-3.7, we have

$$
\begin{aligned}
\|\Gamma(\omega)\|_{\mathcal{X}_{s, \frac{1}{2}}^{\alpha, \theta}} & \leq\left\|W_{0}(t) \phi\right\|_{\mathcal{X}_{s, \frac{1}{2}}^{\alpha, \theta}}+\left\|W_{b d r}(t) \boldsymbol{h}\right\|_{\mathcal{X}_{s, \frac{1}{2}}^{\alpha, \theta}}+\left\|\int_{0}^{t} W_{0}(t-\tau)\left(\omega \omega_{x}\right)(\tau)\right\|_{\mathcal{X}_{s, \frac{1}{2}}^{\alpha, \theta}} \\
& \leq C\|(\phi, h)\|_{D_{s, T}}+C \theta^{\mu}\|\omega\|_{\mathcal{X}_{s, \frac{1}{2}}^{\alpha, \theta}}^{2}
\end{aligned}
$$

Let $\eta=2 C\|(\phi, \boldsymbol{h})\|_{D_{s, T}} \leq 2 C r$ and introduce the ball

$$
B_{\eta}:=\left\{\omega \in \mathcal{X}_{s, b}^{\alpha, \theta}:\|w\|_{\mathcal{X}_{s, \frac{1}{2}}^{\alpha, \theta}} \leq \eta\right\} .
$$

Then for $\omega \in B_{\eta}$,

$$
\begin{aligned}
\|\Gamma(\omega)\|_{\mathcal{X}_{s, \frac{1}{2}}^{\alpha, \theta}} & \leq \eta / 2+C \theta^{\mu} \eta^{2} \\
& \leq \eta / 2+\eta / 2=\eta
\end{aligned}
$$

when we select $T^{*}=\theta>0$ and $2 C\left(T^{*}\right)^{\mu} \eta<1$. Therefore,

$$
\Gamma\left(B_{\eta}\right) \subset B_{\eta} .
$$

Similarly, taking $v, \omega \in \mathcal{X}_{s, \frac{1}{2}}^{T^{*}}$,

$$
\begin{aligned}
\|\Gamma(v)-\Gamma(\omega)\|_{\mathcal{X}_{s, \frac{1}{2}}^{\alpha, T T^{*}}} & \leq C \theta^{\mu}\|v-\omega\|_{\mathcal{X}_{s, \frac{1}{2}}^{\alpha, T^{*}}}\|v+\omega\|_{\mathcal{X}_{s, \frac{1}{2}}^{\alpha, T^{*}}} \\
& \leq C \theta^{\mu}\|v-\omega\|_{\mathcal{X}_{s, \frac{1}{2}}^{\alpha, T^{*}}}\left(\|v\|_{\mathcal{X}_{s, \frac{1}{2}}^{\alpha, T^{*}}}+\|\omega\|_{\mathcal{X}_{s, \frac{1}{2}}^{\alpha, T^{*}}}\right) \\
& \leq 2 \eta C \theta^{\mu}\|v-\omega\|_{\mathcal{X}_{s, \frac{1}{2}}^{T^{*}}} \\
& \leq \beta\|v-\omega\|_{\mathcal{X}_{s, \frac{1}{2}}^{\alpha, T^{*}}}
\end{aligned}
$$

with $\beta=2 C\left(T^{*}\right)^{\mu} \eta<1$ as we have defined before. Then, by the contraction mapping principle, the fixed point $u$ is the unique desired solution of (3.10). The proof is complete.

\section{Concluding Remarks}

The focus of our discussion has been the well-posedness of the Initial-Boundary Value Problem of the KdV equation posed on the finite interval $(0, L)$ :

$$
\left\{\begin{array}{l}
u_{t}+u_{x}+u_{x x x}+u u_{x}=0, \quad x \in(0, L), t>0, \\
u(x, 0)=\phi(x), \\
u(0, t)=h_{1}(t), u_{x}(L, x)=h_{2}(t), u_{x x}(L, t)=h_{3}(t) .
\end{array}\right.
$$

It is considered with the initial data $\phi \in H^{s}(0, L)$ and the boundary data $\boldsymbol{h}=\left(h_{1}, h_{2}, h_{3}\right)$ in the space $D_{s, T}:=H^{\frac{s+1}{3}}(0, T) \times H^{\frac{s}{3}}(0, T) \times H^{\frac{s-1}{3}}(0, T)$ for $s>-\frac{3}{4}$. Using the approaches developed in [5,7] with some modifications we have succeeded in showing that the IBVP (4.1) is locally well-posed in the space $H^{s}(0, L)$ for any $s>-\frac{3}{4}$ with $s \neq \frac{2 j+1}{2}, j=0,1,2, \ldots$, which extends and improves the earlier works of Colin and Ghidaglia [16-18]. 
However, the well-posedness results presented in Theorems 1.4 and 1.5 are conditional in the sense that the uniqueness holds in a Banach space stronger than $C\left([0, T] ; H^{s}(0, L)\right)$. In particular, in the case of $s \geq 0$, according to Theorem 1.4, the uniqueness holds in the space

$$
C\left([0, T] ; H^{s}(0, L)\right) \cap L^{2}\left(0, T ; H^{s+1}(0, L)\right)
$$

rather than in the space $C\left([0, T] ; H^{s}(0, L)\right)$. Also when $-\frac{3}{4}<s<0$, Theorem 1.5 states that the uniqueness holds in the space

$$
C\left([0, T] ; H^{s}(0, L)\right) \cap X_{s, \frac{1}{2}}^{\alpha, T}
$$

which is a stronger subspace of $C\left([0, T] ; H^{s}(0, L)\right)$. A question arises naturally:

Does the uniqueness hold in the space $C\left([0, T] ; H^{s}(0, L)\right)$ ?

If the uniqueness does hold in the space $C\left([0, T] ; H^{s}(0, L)\right)$, then the corresponding well-posedness is called unconditional well-posedness. (The interested readers are referred to Bona et al. [6] for conditional and unconditional well-posedness of evolution equations.)

By using the usual energy estimate method, one can show that the uniqueness does hold for the IBVP (4.1) in the space $C\left([0, T] ; H^{s}(0, L)\right)$ when $s>\frac{3}{2}$. Thus the IBVP $(4.1)$ is unconditionally (locally) well-posed in the space $H^{s}(0, L)$ for any $s>\frac{3}{2}$ with

$$
s \neq \frac{2 j+1}{2}, \quad j=1,2, \ldots
$$

The following question remains open.

Question 4.1. Is the IBVP (4.1) unconditionally well-posed in the space $H^{s}(0, L)$ for some $s<\frac{3}{2}$ ?

By contrast, the IBVP

$$
\left\{\begin{array}{l}
u_{t}+u_{x}+u_{x x x}+u u_{x}=0, \quad x \in(0, L), t>0 \\
u(x, 0)=\phi(x), \\
u(0, t)=h_{1}(t), u(L, x)=h_{2}(t), u_{x}(L, t)=h_{3}(t)
\end{array}\right.
$$

is known to be unconditionally well-posed in the space $H^{s}(0, L)$ for any $s>-1$. This is due to the fact that the IBVP (4.2) is known to be globally well-posed in the space $H^{s}(0, L)$ for any $s \geq 0$. In particular, its classical solutions exist globally. However, the IBVP (4.1) is only known to be locally well-posed. Whether it is globally well-posed is still an open question.

Question 4.2. Is the IBVP (4.1) globally well-posed in the space $H^{s}(0, L)$ for some $s \geq 0$ ?

This is the same question asked earlier by Colin and Ghidaglia [18]. They showed that that if $\phi \in H^{1}(0, L)$ and $h_{j} \in C^{1}\left(\mathbb{R}^{+}\right), j=1,2,3$ are small enough, then the corresponding solution $u$ of (4.1) exists globally:

$$
u \in L^{\infty}\left(\mathbb{R}^{+} ; H^{1}(0, L)\right) .
$$

Recently, Rivas et al. [41] showed that the solutions of the IBVP (4.1) exist globally (in time) in the space $H^{s}(0, L)$ for any $s \geq 0$ as long as its auxiliary data $(\phi, \boldsymbol{h})$ is small in the space $D_{T}^{s}$. In addition, they have shown that those small amplitude solutions decay exponentially if their boundary value $\boldsymbol{h}(t)$ decays exponentially. In particular, those solutions satisfying homogenous boundary conditions decay exponentially in the space $H^{s}(0, L)$ if their initial values are small in $H^{s}(0, L)$.

Note that a positive answer to Question 4.2 leads to a positive answer to Question 4.1 using the general approach developed by Bona et al. [6] for establishing unconditional well-posedness of nonlinear evolution equations. 
Recently, Bona et al. [9] showed that the IBVP (4.2) is locally (unconditionally) well-posed in the space $H^{s}(0, L)$ for any $s>-1$. One of the key steps in their approach is to transfer the IBVP (4.2) of the KdV equation to an equivalent IBVP of the KdV-Burgers equation. Precisely, let

$$
u(x, t)=\mathrm{e}^{2 t-x} v(x, t) .
$$

Then $u$ is a solution of the IBVP (4.2) if and only if $v$ is a solution of the following IBVP of the KdV-Burgers equation posed on the finite interval $(0, L)$ :

$$
\left\{\begin{array}{l}
v_{t}+4 v_{x}-3 v_{x x}+v_{x x x}+\mathrm{e}^{2 t-x}\left(v v_{x}-v^{2}\right)=0, x \in(0, L), t \geq 0, \\
v(x, 0)=\phi(x) \mathrm{e}^{x}, \\
v(0, t)=\mathrm{e}^{-2 t} h_{1}(t), v(L, t)=\mathrm{e}^{-2 t+L} h_{2}(t), v_{x}(L, t)=\mathrm{e}^{-2 t+L} h_{3}(t)+h_{1}(t) \mathrm{e}^{-2 t+L} .
\end{array}\right.
$$

Consequently, one can adapt the approach of Molinet [36] in dealing with the pure initial value problems of the KdV-Burgers equation posed either on the whole line $\mathbb{R}$ or on a periodic domain $\mathbb{T}$ to show that the IBVP (4.3) is locally well-posed in the space $H^{s}(0, L)$ for any $s>-1$. However, the same transformation converts the $\operatorname{IBVP}_{i i}(4.1)$ to the following IBVP of the KdV-Burgers equation

$$
\left\{\begin{array}{l}
v_{t}+4 v_{x}-3 v_{x x}+v_{x x x}+\mathrm{e}^{2 t-x}\left(v v_{x}-v^{2}\right)=0, x \in(0, L), t \geq 0, \\
v(x, 0)=\phi(x) \mathrm{e}^{x} \\
v(0, t)=\mathrm{e}^{-2 t} h_{1}(t), v_{x}(L, t)-v(L, t)=\mathrm{e}^{-2 t+L} h_{2}(t) \\
v_{x x}(L, t)-v(L, t)=\mathrm{e}^{-2 t+L}\left(2 h_{2}(t)+h_{3}(t)\right) .
\end{array}\right.
$$

Note that the boundary conditions of (4.4) are different from those of (4.3). That brings a challenge to show that the IBVP (4.4) to be locally well-posed in $H^{s}(0, L)$ for $s>-1$. The following question thus remains open.

Question 4.3. Is the IBVP well-posed in the space $H^{s}(0, L)$ for $-1<s \leq-\frac{3}{4}$ ?

Finally we would like to point out that the KdV equation including, in particular, the IBVP (4.2) has been extensively studied in the past twenty years from control point of view ( $c f .[14,15,21,33,38,40,42,43,45,46,48,52]$ and the reference therein). The interested readers are specially referred to Rosier and Zhang [44] for a recent survey on this subject. By contrast, the study of the IBVP (4.1) is still widely open. It will be very interesting to see if there are any differences between the IBVP (4.1) and the IBVP (4.2) from control point view.

Acknowledgements. Ivonne Rivas was partially supported by the Taft Memorial Fund at the University of Cincinnati through Graduate Dissertation Fellowship. Bing-Yu Zhang was partially supported by the Taft Memorial Fund at the University of Cincinnati. The work was partially conducted while the second author (IR) and the third author (BZ) were participating the trimester program, Control of Partial Differential Equations and Applications, held at the Institut Henri Poincaré (Paris) from October 1, 2010 to December 18, 2010. They thank the Institute for its hospitality and financial support.

\section{REFERENCES}

[1] T.B. Benjamin, J.L. Bona and J.J. Mahony, Model equations for long waves in nonlinear dispersive systems. Proc. R. Soc. London A 272 (1972) 47-78.

[2] J.L. Bona, W.G. Pritchard and L.R. Scott, An evaluation of a model equation for water waves. Philos. Trans. Roy. Soc. London Ser. A 302 (1981) 457-510.

[3] J.L. Bona, S.M. Sun and B.-Y. Zhang, A nonhomogeneous boundary-value problem for the Korteweg-de Vries equation in a quarter plane. Trans. Amer. Math. Soc. 354 (2002) 427-490.

[4] J.L. Bona, S.M. Sun and B.-Y. Zhang, Forced oscillations of a damped korteweg-de Vries equation in a quarter plane. Commun. Partial Differ. Equ. 5 (2003) 369-400.

[5] J.L. Bona, S.M. Sun and B.-Y. Zhang, A nonhomogeneous boundary-value problem for the Korteweg-de Vries equation on a finite domain. Commun. Partial Differ. Equ. 28 (2003) 1391-1436. 
[6] J.L. Bona, S.M. Sun and B.-Y. Zhang, Conditional and unconditional well posedness of nonlinear evolution equations. Adv. Differ. Equ. 9 (2004) 241-265.

[7] J.L. Bona, S.M. Sun and B.-Y. Zhang, Boundary smoothing properties of the Korteweg-de Vries equation in a quarter plane and applications. Dyn. Partial Differ. Equ. 3 (2006) 1-69.

[8] J.L. Bona, S.M. Sun and B.-Y. Zhang, Nonhomogeneous problems for the Korteweg-de Vries and the Korteweg-de VriesBurgers equations in a quarter plane. Ann. Henri Poincaré 25 (2008) 1145-1185.

[9] J.L. Bona, S.M. Sun and B.-Y. Zhang, Nonhomogeneous problem for the Korteweg-de Vries equation in a bounded domain II. J. Differ. Equ. 247 (2009) 2558-2596.

[10] J. Bourgain, Fourier transform restriction phenomena for certain lattice subsets and applications to nonlinear evolution equations, part I: Shrödinger equations. Geom. Funct. Anal. 3 (1993) 107-156.

[11] J. Bourgain, Fourier transform restriction phenomena for certain lattice subsets and applications to nonlinear evolution equations, part II: the KdV-equation. Geom. Funct. Anal. 3 (1993) 209-262.

[12] B.A. Bubnov, Generalized boundary value problems for the Korteweg-de Vries equation in bounded domain. Differ. Equ. 15 (1979) 17-21.

[13] B.A. Bubnov, Solvability in the large of nonlinear boundary-value problem for the Korteweg-de Vries equations. Differ. Equ. 16 (1980) 24-30.

[14] E. Cerpa, Exact controllability of a nonlinear Korteweg-de Vries equation on a critical spatial domain. SIAM J. Control Optim. 46 (2007) 877-899.

[15] E. Cerpa and E. Crépeau, Boundary controllability for the nonlinear Korteweg-de Vries equation on any critical domain. Ann. Henri Poincaré 26 (2009) 457-475.

[16] T. Colin and J.-M. Ghidaglia, Un problème aux limites pour l'équation de Korteweg-de Vries sur un intervalle borné (French) [A boundary value problem for the Korteweg-de Vries equation on a bounded interval] Journées équations aux Drives Partielles, Saint-Jean-de-Monts, Exp. No. III, École Polytech., Palaiseau (1997), p. 10.

[17] T. Colin and J.-M. Ghidaglia, Un problème mixte pour l'équation de Korteweg-de Vries sur un intervalle borné (French) [A mixed initial-boundary value problem for the Korteweg-de Vries equation on a bounded interval]. C. R. Acad. Sci. Paris Sér. I Math. 324 (1997) 599-603.

[18] T. Colin and J.-M. Ghidaglia, An initial-boundary-value problem fo the Korteweg-de Vries equation posed on a finite interval. Adv. Differ. Equ. 6 (2001) 1463-1492.

[19] T. Colin and M. Gisclon, An initial-boundary-value problem that approximate the quarter-plane problem for the Korteweg-de Vries equation. Nonlinear Anal. 46 (2001) 869-892.

[20] J.E. Colliander and C. Kenig, The generalized Korteweg-de Vries equation on the half line. Commun. Partial Differ. Equ. 27 (2002) 2187-2266.

[21] J.-M. Coron and E. Crépeau, Exact boundary controllability of a nonlinear KdV equation with a critical length. J. Eur. Math. Soc. 6 (2004) 367-398.

[22] A.V. Faminskii, On an initial boundary value problem in a bounded domain for the generalized Korteweg-de Vries equation, International Conference on Differential and Functional Differential Equations (Moscow, 1999). Funct. Differ. Equ. 8 (2001) $183-194$.

[23] A.V. Faminskii, Global well-posedness of two initial-boundary-value problems for the Korteweg-de Vries equation. Differ. Integral Equ. 20 (2007) 601-642.

[24] J.-M. Ghidaglia, Weakly damped forced Korteweg-de Vries equations behave as a finite-dimensional dynamical system in the long time. J. Differ. Equ. 74 (1988) 369-390.

[25] J.-M. Ghidaglia, A note on the strong convergence towards attractors of damped forced KdV equations. J. Differ. Equ. 110 (1994) 356-359.

[26] J. Holmer, The initial-boundary value problem for the Korteweg-de Vries equation. Commun. Partial Differ. Equ. 31 (2006) 1151-1190.

[27] T. Kappeler and P. Topalov, Global well-posedness of KdV in $H^{-1}(T, R)$. Duke Math. J. 135 (2006) 327-360.

[28] T. Kato, On the Korteweg-de Vries equation. Manuscr. Math. 28 (1979) 89-99.

[29] T. Kato, On the Cauchy problem for the (generalized) Korteweg-de Vries equations. Advances in Mathematics Supplementary Studies, Stud. Appl. Math. 8 (1983) 93-128.

[30] C. Kenig, G. Ponce and L. Vega, Well-posedness of the initial value problem for the Korteweg-de Vries equation. J. Amer. Math. Soc. 4 (1991) 323-347.

[31] C. Kenig, G. Ponce and L. Vega, Well-posedness and scattering results for the generalized Korteweg-de Vries equations via the contraction principle. Commun. Pure Appl. Math. 46 (1993) 527-620

[32] C. Kenig, G. Ponce and L. Vega, A bilinear estimate with applicatios to the KdV equation. J. Amer. Math. Soc. 9 (1996) 573-603.

[33] V. Komornik, D.L. Russell and B.-Y. Zhang, Stabilization de l'equation de Korteweg-de Vries. C. R. Acad. Sci. Paris 312 (1991) 841-843.

[34] E.F. Kramer and B.-Y. Zhang, Nonhomogeneous boundary value problems for the Korteweg-de Vries equation on a bounded domain. J. Syst. Sci. Complex 23 (2010) 499-526.

[35] L. Monilet, A note on ill-posedness for the KdV equation. Differ. Integral Equ. 24 (2011) 759-765.

[36] L. Molinet and F. Ribaud, On the low regularity of the Korteweg-de Vries-Burgers equation. Int. Math. Res. Not. (2002) 1979-2005. 
[37] L. Molinet and S. Vento, Sharp ill-posedness and well-posedness results for the KdV-Burgers equation: the periodic case. arXiv: 10054805V1 [Math AP] (2010).

[38] A.F. Pazoto, Unique continuation and decay for the Korteweg-de Vries equation with localized damping. ESAIM: COCV 11 (2005) 473-486.

[39] A. Pazy, Semigroups of linear operators and applications to partial differential equations. Appl. Math. Sci. 44 (1983).

[40] G. Perla-Menzala, C.F. Vasconcellos and E. Zuazua, Stabilization of the Korteweg-de Vries equation with localized damping. Q. Appl. Math. 60 (2002) 111-129.

[41] I. Rivas, M. Usman and B.-Y. Zhang, Global well-posedness and asymptotic behavior of a class of initial-boundary-value problem of the Korteweg-de Vries equation on a finite domain. Math. Control Rel. Fields 1 (2011) 61-81.

[42] L. Rosier, Exact boundary controllability for the Korteweg-de Vries equation on a bounded domain. ESAIM: COCV 2 (1997) $33-55$.

[43] L. Rosier and B.-Y. Zhang, Global stabilization of the generalized Korteweg-de Vries equation. SIAM J. Control Optim. 45 (2006) 927-956.

[44] L. Rosier and B.-Y. Zhang, Control and stabilization of the Korteweg-de Vries equation: recent progresses J. Syst. Sci. Complex. 22 (2009) 647-682.

[45] D.L. Russell and B.-Y. Zhang, Controllability and stabilizability of the third-order linear dispersion equation on a periodic domain. SIAM J. Control Optim. 31 (1993) 659-676.

[46] D.L. Russell and B.-Y. Zhang, Smoothing and decay properties of solutions of the Korteweg-de Vries equation on a periodic domain with point dissipation. J. Math. Anal. Appl. 190 (1995) 449-488.

[47] L. Tartar, Interpolation non linéaire et régularité. J. Funct. Anal. 9 (1972) 469-489.

[48] B.-Y. Zhang, Boundary stabilization of the Korteweg-de Vries equations, Proc. of International Conference on Control and Estimation of Distributed Parameter Systems: Nonlinear Phenomena. Vorau, Styria, Austria (1993). International Series of Numer. Math. 118 (1994) 371-389.

[49] B.-Y. Zhang, A remark on the Cauchy problem for the Korteweg de-Vries equation on a periodic domain. Differ. Integral Equ. 8 (1995) 1191-1204.

[50] B.-Y. Zhang, Analyticity of solutions for the generalized Korteweg de-Vries equation with respect to their initial datum. SIAM J. Math. Anal. 26 (1995) 1488-1513.

[51] B.-Y. Zhang, Taylor series expansion for solutions of the Korteweg-de Vries equation with respect to their initial values. J. Func. Anal. 129 (1995) 293-324.

[52] B.-Y. Zhang, Exact boundary controllability of the Korteweg-de Vries equation. SIAM J. Control Optim. 37 (1999) 543-565. 